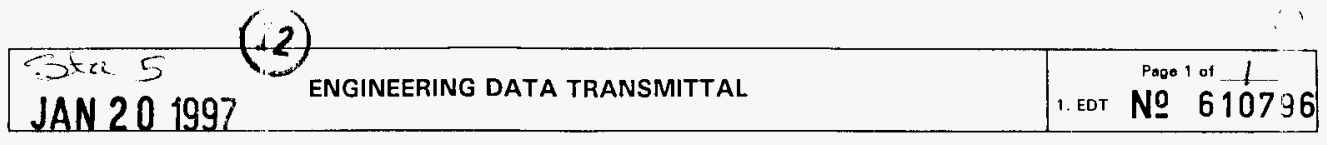

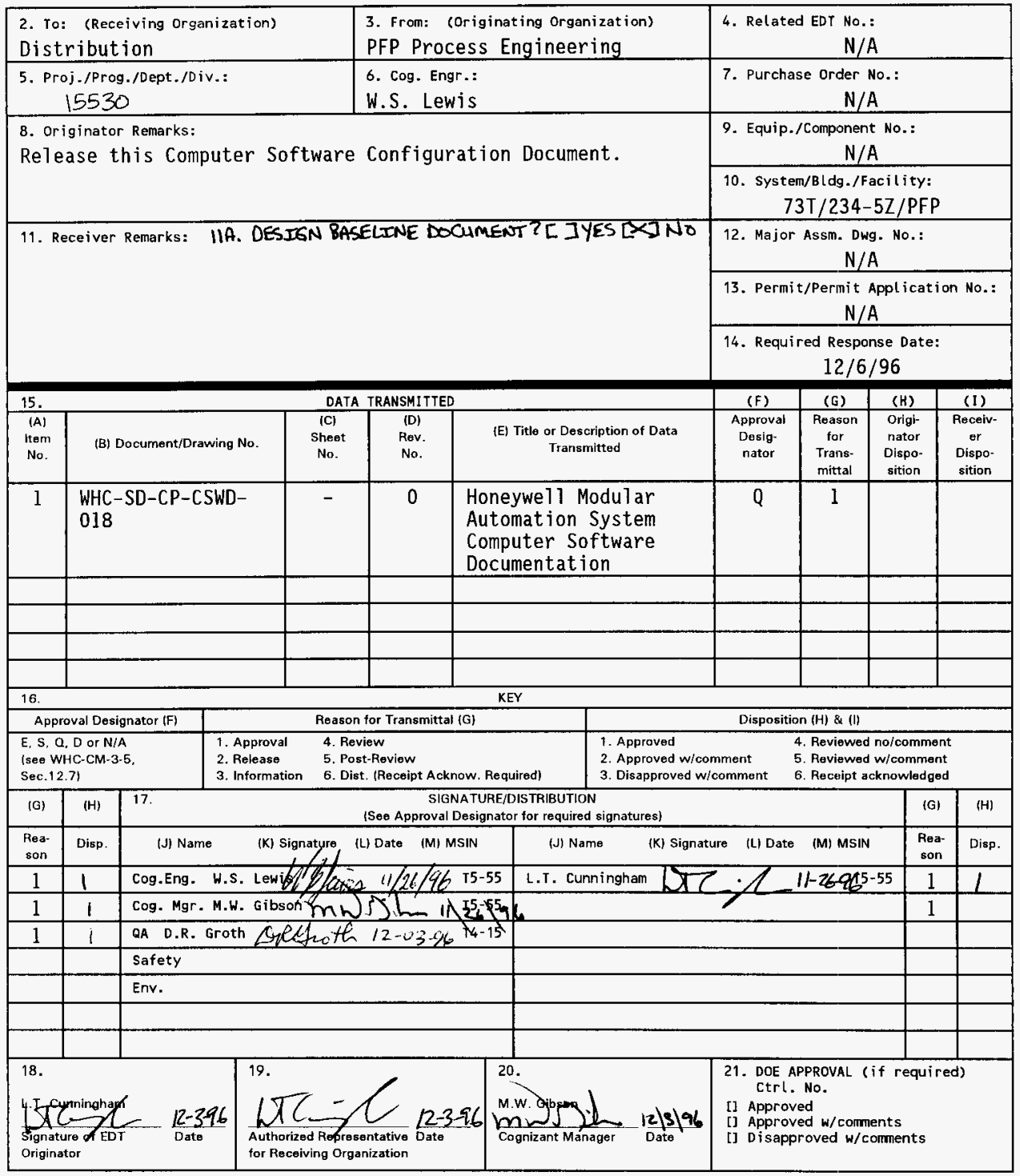




\section{HONEYWELL MODULAR AUTOMATION SYSTEM COMPUTER SOFTWARE DOCUMENTATION}

\section{L.T. Cunningham}

B \& W Hanford Co. , Richland. WA 99352

U.S. Department of Energy Contract DE-AC06-87RL10930

$\begin{array}{llll}\text { EDT/ECN: } & 610796 & \text { UC: UC-506 } & \\ \text { Org Code: } & 15530 & \text { Charge Code: } & \text { K6134 } \\ \text { B\&R Code: } & \text { EW7003000 } & \text { Total Pages: } 111\end{array}$

Key Words: Honeywe11. Modular Automation System, Computer Software Management Plan

Abstract: This document provides a Computer Software Documentation for a new Honeywe11 Modular Automation System (MAS) being installed in the Plutonium Finishing Plant (PFP). This system will be used to control new thermal stabilization furnaces in HA-21I.

Honeywel1 is registered trademark of Honeywell Inc., Minneapolis. MN. Intellution and FIX DMACS are registered trademarks of Intellution. Inc. INTEL and Pentium are registered trademarks of INTEL Corp. Microsoft Windows and Excel are registered trademarks of Microsoft Corp.

TRADEMARK DISCLAIMER. Reference herein to any specific commercial product, process, or service by trade name, trademark, manufacturer, or otherwise, does not necessarily constitute or imply its endorsement. recommendation, or favoring by the United States Government or any agency thereof or its contractors or subcontractors.

Printed in the United States of America. To obtain copies of this document, contact: WHC/BCS Document Control Services, P.0. Box 1970. Mailstop H6-08. Richland WA 99352, Phone (509) 372-2420; Fax (509) 376-4989.
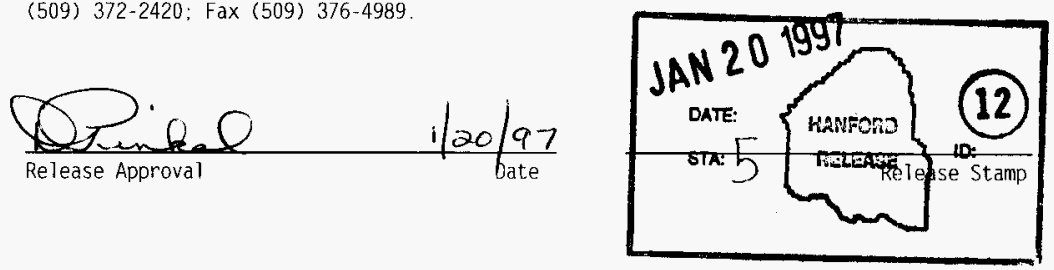

Approved for Public Release 
TABLE OF CONTENTS

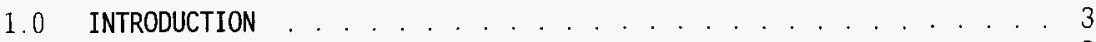

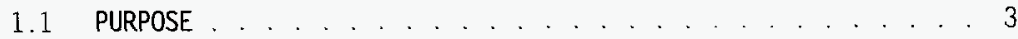

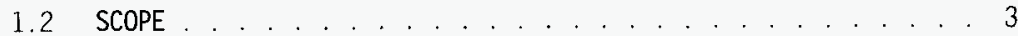

2.0 SYSTEM HARDWARE DESCRIPTION . . . . . . . . . . . . . . . . . . . 3

2.0 SYSTEM HARDWARE DESCRIPTION . . . . . . . . . . . . . . . . . . 4

3.0 SYSTEM SOFTWARE CONFIGURATION FILES . . . . . . . . . . . . . . . . . 4

4.0 GRAPHICAL FILES . . . . . . . . . . . . . . . . . 5

5.0 TAG GROUP FILES . . . . . . . . . . . . . . 5

APPENDIX A - CCC BLOCK DIAGRAMS AND REPORTS . . . . . . . . . . . . 6

APPENDIX B - PCS LADDER LOGIC . . . . . . . . . . . . . . 47

APPENDIX C - SET POINT PROGRAMS . . . . . . . . . . . . 50

APPENDIX D - GRAPHIC DISPLAYS AND REPORTS . . . . . . . . . . . 57

APPENDIX E - TAG GROUP REPORTS . . . . . . . . . . . 108 


\subsection{INTRODUCTION}

\subsection{PURPOSE}

The purpose of this Computer Software Document (CSWD) is to provide configuration control of the Honeywell Modular Automation System (MAS) in use at the Plutonium Finishing Plant (PFP). The Honeywell MAS controls thermal stabilization furnaces in HA-21I.

\subsection{SCOPE}

This CSWD describes hardware and PFP developed software for control of stabilization furnaces installed into HA-21I. Copies of reports generated by the Honeywell software for the PFP developed software have been attached as addendums.

This plan applies to PFP Process Engineering Cognizant Manager and Stabilization Cognizant Engineers responsible for the Honeywel1 MAS software/hardware and administration of the Honeywell System.

\subsection{SYSTEM HARDWARE DESCRIPTION}

The Honeywell MAS consists of one PC work station, a "cable link." and the process control rack. Connections from furnaces in HA-21I connected to modules in the process control rack.

The work station is a DELL personal computer with an INTEL ${ }^{\circledast}$ Pentium ${ }^{\circledR}$ processor and operates in a Microsoft ${ }^{\circledR}$ Windows $^{\text {TM }}$ environment. Avai lable software includes the Honeywe11 PC supervisor, Intellution ${ }^{\circledR}$ FIX DMACS ${ }^{\text {IM }}$.

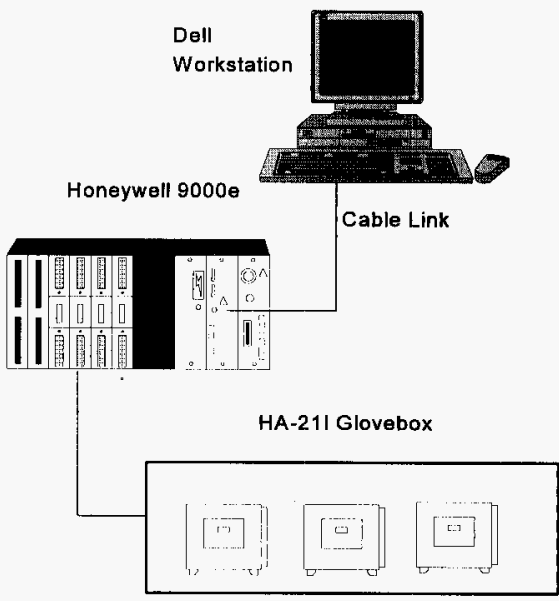
and Microsoft ${ }^{\boxplus}$ Windows $^{\mathrm{TM}}$ and Exce $]^{T M}$.

The PC work station is connected to the PLC with a "cable link" (coaxial cable). Each end of cable is terminated with 50 -ohm resistors. 
The process control rack contains the following modules: a Model 9000e logic control processor (9010-012), a 15-amp power supply (620-0083), an Ethernet Loop Processor Module (620-0073), two universal analog input modules (621-0020R), two analog output modules (621-0010AR), discrete input module (621-1160R), and a discrete output module (621-2150R). Two open slots will remain in the control rack for future expansion.

The universal analog input modules (621-0020R) can each accept 16 inputs. Input addresses 32 to 43 are used as thermocouple inputs for furnace control temperature, furnace high temperature, furnace off-gas temperature, and HA-21I glovebox temperature. These inputs are configured as Type $K$ thermocouples.

Each analog output module (621-0010AR) is capable of four output signals. Addresses 72 to 74 are configured to output 4-20mA control signals to Silicon Control Rectifiers (SCR).

The 621-1160R discrete input module accepts 16 inputs. Addresses 80 to 82 accept input from furnace door limit switches and addresses 83 and 84 provide power indication for HA-21I furnace off-gas faris.

The 621-2150R discrete output module has 16 outputs. Some outputs wi11 allow relays to energize and permit power to furnaces heating elements. These relays are an integral part to the interlock schemes for powering the furnaces. Other outputs will be used to start and stop HA-21I furnace off-gas fans.

\subsection{SYSTEM SOFTWARE CONFIGURATION FILES}

The continuous control chart (CCC) is the configuration software file for the 59000 Controller that creates loop control graphically through the PC Supervisor. Addresses and point names are used to define source and destination of data within the CCC configuration. These addresses identify physical connection points for field wiring to I/O modules plus internal I/0 and registers with the relay ladder logic. A copy of the CCC block diagram and report for thermal stabilization furnaces are attached as Appendix $A$. Reports include control block configurations and point configurations input by programmer.

The PCS Relay Ladder Logic allows the programmer to define discrete control functions in the control strategy with logic elements such as coils and relays. A printout of the Ladder Logic is attached as Appendix B. 


\subsection{GRAPHICAL FILES}

These files provide graphical representation of the thermal stabilization process. The operator will start from the Furnace Overview screen. From the overview screen, operators can get to individual furnace screens by clicking on a furnace. Operators will have increased monitoring abilities and will be able to start or stop the selected individual furnace from this screen. The Off-Gas Fan Control screen is accessible from the overview screen by clicking on OFF-GAS FAN. Each of the off-gas fans can be started or stopped with this graphic. Screens to monitor glovebox temperature, furnace temperatures, and current alarms are also available from the overview screen. Copies of these graphics are attached in Appendix $\mathrm{C}$ with corresponding computer generated report.

\subsection{TAG GROUP FILES}

The tag group files are sets of tagnames used in a common graphical displays to replace generic tagnames with specific data points. Tag group reports have been generated by the Tag Group Editor to an ASCII file. Copies of these reports are attached in Appendix $D$. 
WHC-SD-CP-CSWD-018, Rev. 0

APPENDIX A

CCC BLOCK DIAGRAMS AND REPORTS 
WHC-SD-CP-CSWD-018, Rev. 0

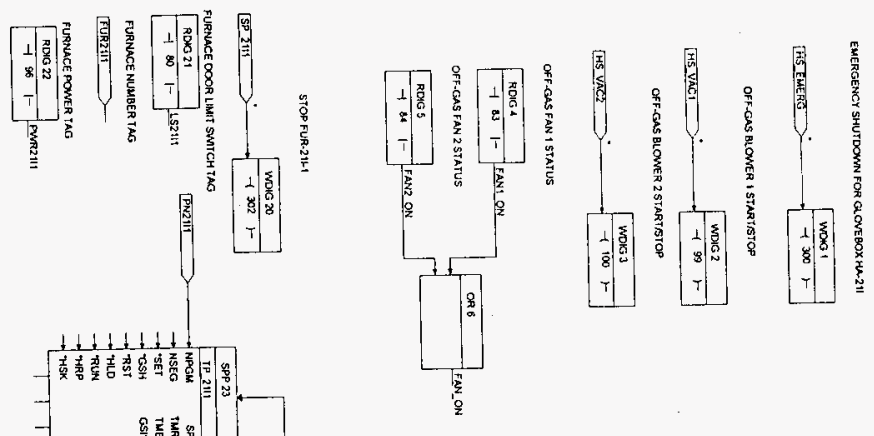

$?$

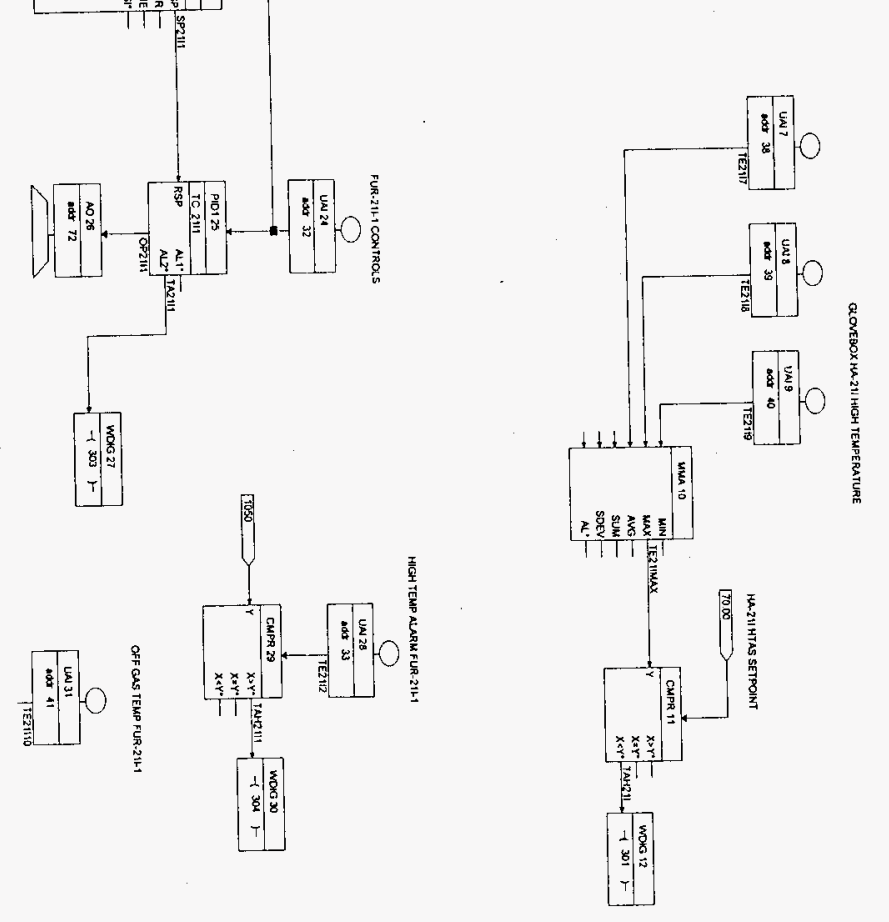


WHC-SD-CP-CSWD-018, Rev. 0

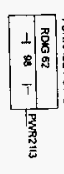$$
\frac{1}{1}
$$

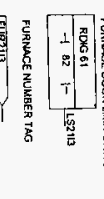
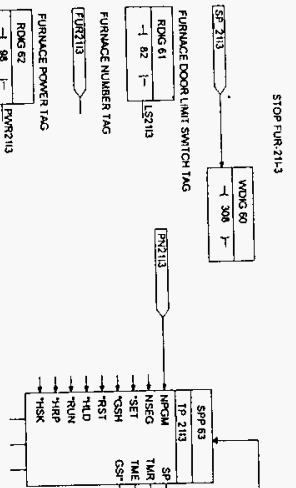

ग1
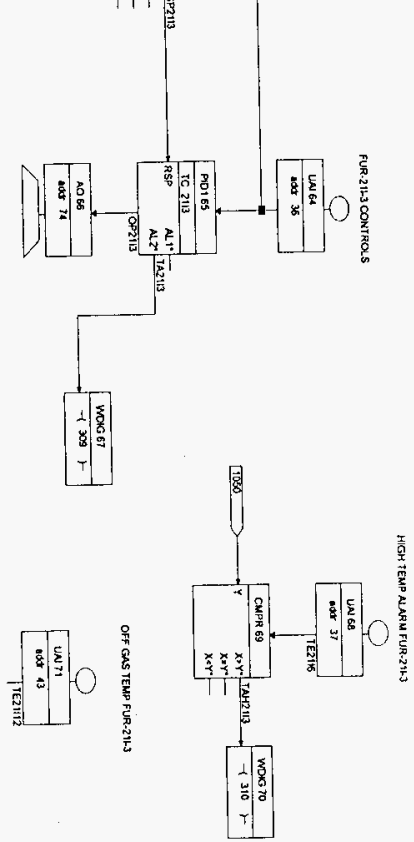
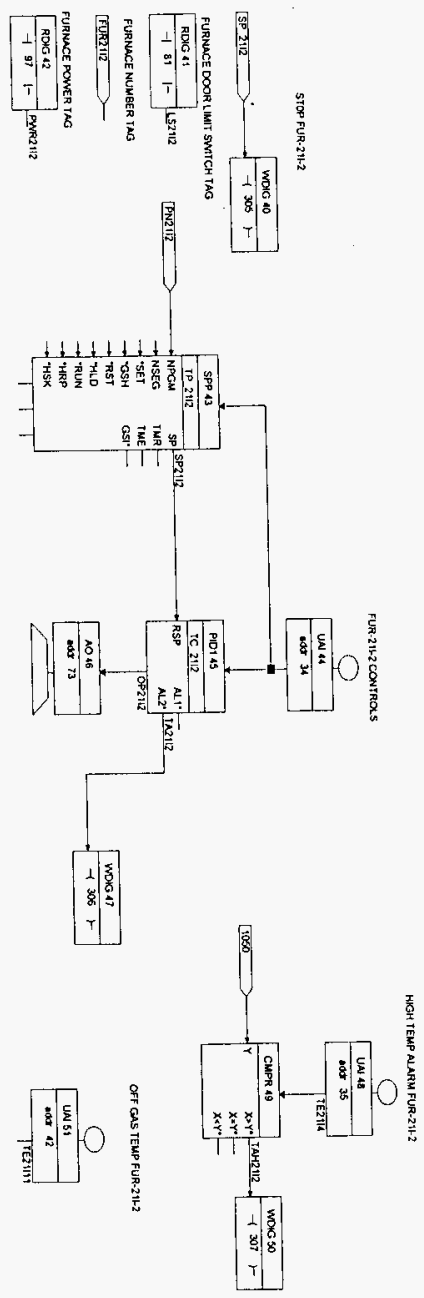
Control Block Configuration - FURN21I

PAGE 1

Nov 06 $199608: 43 \quad$ AM

Block Number: 1

Block Type: WDIG

$\frac{\text { Input Label }}{* \mathrm{X}} \quad \underline{\text { Block }} \quad \begin{gathered}\text { (connected to) } \\ \text { TYpe }\end{gathered}$ output $\frac{\text { Tagname }}{\text { HS_EMERG }}$

Configuration Parameter $\frac{\text { Value }}{300}$

Block Number: 2

Block Type: WDIG

$\frac{\text { Input Label }}{\text { *X Block }} \begin{gathered}\text { (connected to) } \\ \text { Type }\end{gathered}$ output $\frac{\text { Tagname }}{\text { HS_VACI }}$

Configuration Parameter Value

ADDRESS $\frac{99}{9}$

Block Number: 3

Block Type: WDIG

$\frac{\text { Input Label }}{\star \mathrm{X}} \quad \underline{\text { Block }} \begin{gathered}\text { (connected to) } \\ \text { Type }\end{gathered}$ Output $\frac{\text { Tagname }}{\text { HS_VAC2 }}$

Configuration Parameter Value ADDRESS $\frac{100}{100}$

Block Number: 4

Block Type: RDIG

Configuration Parameter Value

ADDRESS 83

Block Number: 5

Block TYpe: RDIG

Configuration Parameter Value

ADDRESS $\frac{\text { C4 }}{84}$ 
Control Block Configuration - FURN21I

PAGE $\quad 2$ Nov $06 \quad 1996 \quad 08: 43$ AM

Block Number: 6

Block Type: OR

$\begin{array}{lcccc}\text { Input Label } & \text { Block } & \begin{array}{c}\text { (connected to) } \\ \text { Type }\end{array} & \begin{array}{l}\text { Output } \\ \text { OUT }\end{array} & \frac{\text { Tagname }}{\text { FAN1_ON }} \\ \star \mathrm{X} & \frac{\text { RDIG }}{5} & \text { RDIG } & \text { OUT* } & \text { FAN2_ON }\end{array}$

Block Number: 7

Block Type: UAI

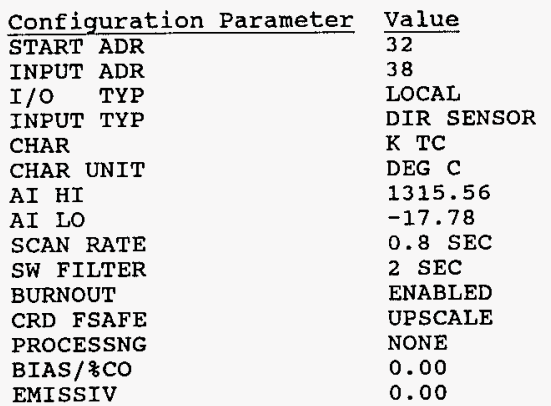


Control Block Configuration - FURN21I

PAGE 3

Nov $061996 \quad 08: 43$ AM

Block Number: 8

Block Type: UAI

\begin{tabular}{|c|c|c|}
\hline Configuration & Parameter & Value \\
\hline START ADR & & $\overline{32}$ \\
\hline INPUT ADR & & 39 \\
\hline I/O TYP & & LOCAL \\
\hline INPUT TYP & & DIR SENSOR \\
\hline CHAR & & $\mathrm{K}$ TC \\
\hline CHAR UNIT & & DEG C \\
\hline AI HI & & 1315.56 \\
\hline AI LO & & -17.78 \\
\hline SCAN RATE & & $0.8 \mathrm{SEC}$ \\
\hline SW FILTER & & $2 \mathrm{SEC}$ \\
\hline BURNOUT & & ENABLED \\
\hline CRD FSAFE & & UPSCALE \\
\hline PROCESSNG & & NONE \\
\hline BIAS / \&CO & & 0.00 \\
\hline EMISSIV & & 0.00 \\
\hline
\end{tabular}

Block Number: 9

Block Type: UAI

\begin{tabular}{ll} 
Configuration Parameter & Value \\
\cline { 1 - 2 } START ADR & 32 \\
INPUT ADR & 40 \\
I/O TYP & LOCAI \\
INPUT TYP & DIR SENSOR \\
CHAR & K TC \\
CHAR UNIT & DEG C \\
AI HI & 1315.56 \\
AI LO & -17.78 \\
SCAN RATE & 0.8 SEC \\
SW FILTER & 2 SEC \\
BURNOUT & ENABLED \\
CRD FSAFE & UPSCALE \\
PROCESSNG & NONE \\
BIAS/\%CO & 0.00 \\
EMISSIV & 0.00
\end{tabular}


Control Block Configuration - FURN21I

PAGE 4

NOV $06 \quad 1996 \quad 08: 43$ AM

Block Number: 10

Block Type: MMA

\begin{tabular}{|c|c|c|c|c|}
\hline 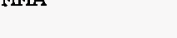 & & (connected & to) & \\
\hline Input Label & Block & Type & output & Tagname \\
\hline$\overline{\mathrm{X} 1}$ & 9 & $\overline{\mathrm{UAI}}$ & $\overline{\text { OUT }}$ & TE21I9 \\
\hline $\mathrm{x} 2$ & 8 & UAI & OUT & TE21I8 \\
\hline $\mathrm{x} 3$ & 7 & UAI & OUT & TE21I7 \\
\hline
\end{tabular}

Block Number: 11

Block Type: CMPR

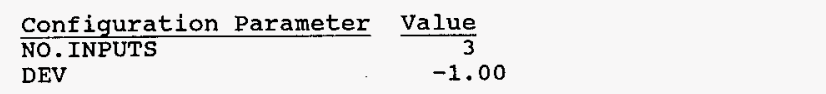

\section{1}

\begin{tabular}{|c|c|c|c|c|}
\hline Input Label & Block & $\begin{array}{l}\text { (connected } \\
\text { Type }\end{array}$ & $\begin{array}{l}\text { to) } \\
\text { output }\end{array}$ & Tagnam \\
\hline & & & -1 & .00 \\
\hline
\end{tabular}

Block Number: 12

Block Type: WDIG

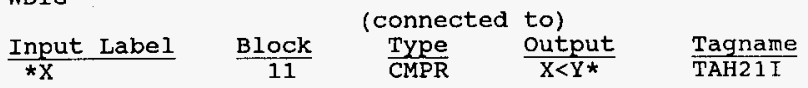

Configuration Parameter Value

ADDRESS $\frac{\text { Co1 }}{301}$

Block Number: 20

Block Number: 20
Block Type:

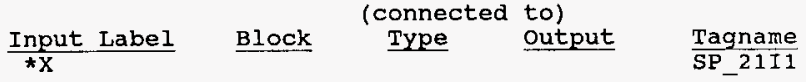

$\frac{\text { Configuration Parameter }}{\text { ADDRESS }} \frac{\text { Value }}{302}$ 
Control Block Configuration - FURN21I NoV $061996 \quad 08: 43$ AM

$\underline{\text { PAGE } 5}$

Block Number: 21

Block Type: RDIG

Configuration Parameter $\frac{\text { Value }}{80}$

Block Number: 22

Block Type: RDIG

Configuration Parameter value

ADDRESS $-\frac{\text { Co }}{96}$

Block Number: 23

Block Type: SPP

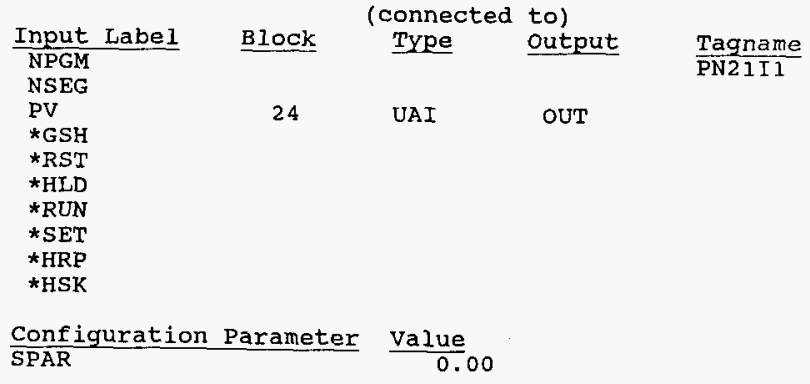


WHC-SD-CP-CSWD-018, Rev. 0

Control Block Configuration - FURN21I

PAGE 6

Nov $06199608: 43$ AM

Block Number: 24

Block Type: UAI

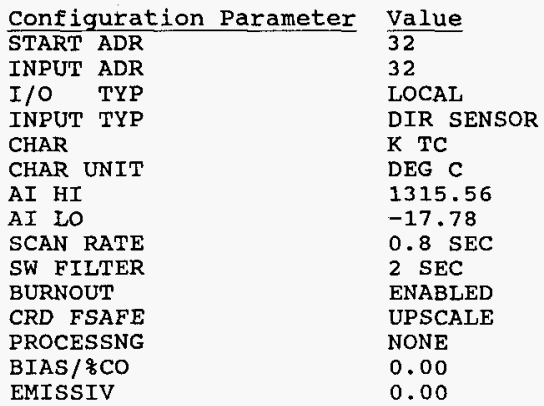


Control Block Configuration - FURN21I $\underline{\text { PAGE } \quad 7}$ Nov $061996 \quad 08: 43$ AM

Block Number: 25

Block Type: PID1

\begin{tabular}{lrrrl} 
Input Label & Block & $\begin{array}{c}\text { (connected to) } \\
\text { Type }\end{array}$ & $\frac{\text { output }}{\text { OUT }}$ & Tagname \\
\cline { 1 - 3 } & $\frac{\text { UAI }}{23}$ & SPP & SP & SP21I1
\end{tabular}

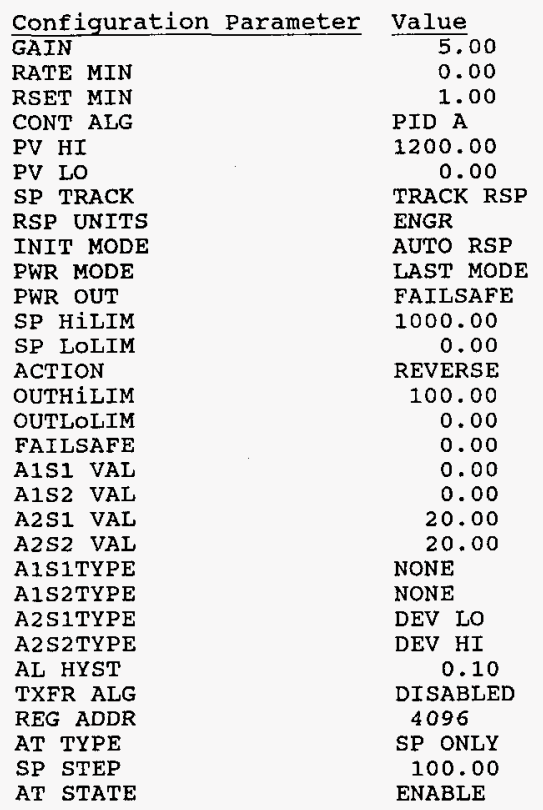


Control Block Configuration - FURN21I

PAGE $\quad 8$

Nov $061996 \quad 08: 43$ AM

Block Number: 26

Block Type: AO

$\frac{\text { Input Label }}{\mathrm{X}} \quad \frac{\text { Block }}{25} \quad \begin{gathered}\text { (connected to) } \\ \frac{\text { Type }}{\text { PID1 }}\end{gathered} \frac{\text { Output }}{\text { OUT }} \quad \frac{\text { Tagname }}{\text { OP21I1 }}$

Configuration Parameter Value

ADDRESS

$\mathrm{X}$ HI

100.00

$\begin{array}{ll}X \text { LO } & 0.00\end{array}$

Block Number: 27

Block Type: WDIG

$\begin{array}{lcc}\text { Input Label } & \frac{\text { Block }}{25} & \begin{array}{c}\text { (connec } \\ \text { Type }\end{array} \\ \text { Configuration Parameter } & \frac{\text { Value }}{303}\end{array}$

Block Number: 28

Block Type: UAI

\begin{tabular}{ll} 
Configuration Parameter & Value \\
\cline { 1 - 2 } START ADR & 32 \\
INPUT ADR & 33 \\
I/O TYP & LOCAL \\
INPUT TYP & DIR SENSOR \\
CHAR NTIT & K TC \\
CHAR UNIT & DEG C \\
AI HI & 1315.56 \\
AI IO & -17.78 \\
SCAN RATE & 0.8 SEC \\
SW FILTER & 2 SEC \\
BURNOUT & ENABLED \\
CRD FSAFE & UPSCALE \\
PROCESSNG & NONE \\
BIAS/\%CO & 0.00 \\
EMISSIV & 0.00
\end{tabular}


Control Block configuration - FURN21I

Nov 061996 08:43 AM

Block Number: 29

Block TYpe: CMPR

$\frac{\text { Input Label }}{\mathrm{X}} \quad \frac{\text { Block }}{28} \quad \frac{\text { Type }}{\mathrm{Y}} \quad \frac{\text { Output }}{\text { OUT }} \frac{\text { Tagname }}{\text { TE21I2 }}$

Block Number: 30

Block Type: WDIG

$\frac{\text { Input Label }}{* X} \quad \frac{\text { Block }}{29} \quad \frac{\text { Type }}{\text { CMPR }} \frac{\text { Output }}{X>Y^{*}} \quad \frac{\text { Tagname }}{\text { TAH2III }}$

Configuration Parameter Value ADDRESS

Block Number: 31

Block Type: UAI

$\begin{array}{ll}\text { Configuration Parameter } & \text { Value } \\ \text { START ADR } & 32 \\ \text { INPUT ADR } & 41 \\ \text { I/O TYP } & \text { LOCAL } \\ \text { INPUT TYP } & \text { DIR SENSOR } \\ \text { CHAR } & \text { K TC } \\ \text { CHAR UNIT } & \text { DEG C } \\ \text { AI HI } & 1315.56 \\ \text { AI LO } & -17.78 \\ \text { SCAN RATE } & 0.8 \text { SEC } \\ \text { SW FILTER } & 2 \text { SEC } \\ \text { BURNOUT } & \text { ENABLED } \\ \text { CRD FSAFE } & \text { UPSCALE } \\ \text { PROCESSNG } & \text { NONE } \\ \text { BIAS/ FCO } & 0.00 \\ \text { EMISSIV } & 0.00\end{array}$

Block Number: 40

Block Type: WDIG

Input Label
$\star \mathrm{X}$

Configuration Parameter value

ADDRESS $\frac{305}{30}$ 
WHC-SD-CP-CSWD-018, Rev. 0

Control Block Configuration - FURN21I

NoV 061996 08:43 AM

PAGE 10

Block Number: 41

Block Type: RDIG

Configuration Parameter value

Block Number: 42

Block Type: RDIG

Configuration Parameter value

ADDRESS

97

Block Number: 43

Block Type: SPP

$\begin{array}{lcccc}\text { Input Label } & \text { Block } & \begin{array}{c}\text { (connected to) } \\ \text { Type }\end{array} & \begin{array}{l}\text { Output } \\ \text { NPGM }\end{array} & \frac{\text { Tagname }}{\text { PN21 I } 2} \\ \text { NSEG } & & & \text { OUT } \\ \text { PV } & 44 & \text { UAI } & \\ \text { *GSH } & & & \\ \text { *RST } & & & \\ \text { *HLD } & & \\ \text { *RUN } & & \\ \text { *SET } & & \\ \text { *HRP } & & \\ \text { *HSK } & & \\ \text { Configuration Parameter } & \frac{\text { Value }}{0.00}\end{array}$


Control Block Configuration - FURN21I

PAGE 11 Nov 061996 08:43 AM

Block Number: 44

Block Type: UAI

$\begin{array}{lll}\text { Configuration Parameter } & & \text { Value } \\ \text { START ADR } & 32 \\ \text { INPUT ADR } & 34 \\ \text { I/O TYP } & \text { LOCAL } \\ \text { INPUT TYP } & \text { DIR SENSOR } \\ \text { CHAR } & \text { K TC } \\ \text { CHAR UNIT } & \text { DEG C } \\ \text { AI HI } & 1315.56 \\ \text { AI LO } & -17.78 \\ \text { SCAN RATE } & 0.8 \text { SEC } \\ \text { SW FIITER } & 2 \text { SEC } \\ \text { BURNOUT } & \text { ENABLED } \\ \text { CRD FSAFE } & \text { UPSCALE } \\ \text { PROCESSNG } & \text { NONE } \\ \text { BIAS/\&CO } & 0.00 \\ \text { EMISSIV } & 0.00\end{array}$


Control Block Configuration - FURN21I

PAGE 12

Nov $06199608: 43$ AM

Block Number: 45

Block TYpe: PIDI

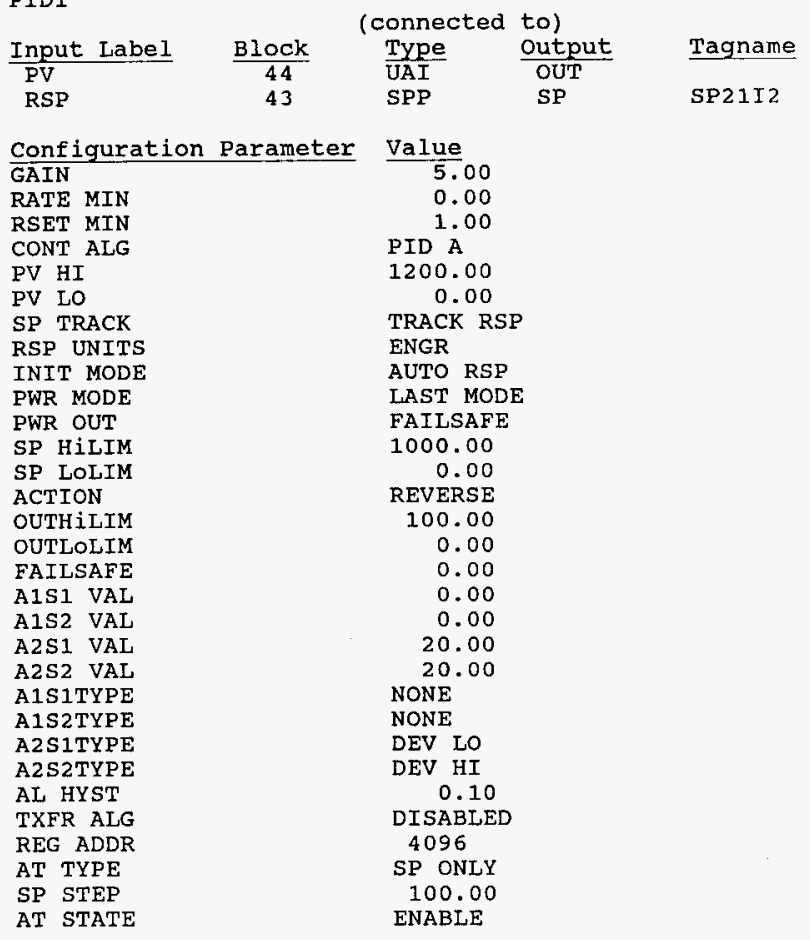


Control Block Configuration - FURN21I

PAGE 13

Nov 06 $1996 \quad 08: 43 \quad$ AM

Block Number: 46

Block Type: AO

$\frac{\text { Input Label }}{\mathrm{X}} \quad \frac{\text { Block }}{45} \quad \frac{\begin{array}{c}\text { (connected to) } \\ \text { Type }\end{array}}{\text { PID1 }} \frac{\text { Output }}{\text { OUT }} \frac{\text { Tagname }}{\text { OP21I }}$

Configuration Parameter $\frac{\text { Value }}{73}$

$\mathrm{X}$ HI

100.00

$X$ LO

0.00

Block Number: 47

Block Type: WDIG

$\frac{\text { Input Label }}{* \mathrm{X}} \quad \frac{\text { Block }}{45} \quad \frac{\begin{array}{c}\text { (connected to) } \\ \text { Type }\end{array}}{\text { PID1 }} \frac{\text { Output }}{\text { AL2* }} \quad \begin{gathered}\text { Tagname } \\ \text { TA21I2 }\end{gathered}$

$\frac{\text { Configuration Parameter }}{\text { ADDRESS }} \frac{\text { Value }}{306}$

ADDRESS

Block Number: 48

Block Type: UAI

$\begin{array}{ll}\text { Configuration Parameter } & \text { Value } \\ \text { START ADR } & 32 \\ \text { INPUT ADR } & 35 \\ \text { I/O TYP } & \text { LOCAL } \\ \text { INPUT TYP } & \text { DIR SENSOR } \\ \text { CHAR N } & \text { K TC } \\ \text { CHAR UNIT } & \text { DEG C } \\ \text { AI HI } & 1315.56 \\ \text { AI LO } & -17.78 \\ \text { SCAN RATE } & 0.8 \text { SEC } \\ \text { SW FILTER } & 2 \text { SEC } \\ \text { BURNOUT } & \text { ENABLED } \\ \text { CRD FSAFE } & \text { UPSCALE } \\ \text { PROCESSNG } & \text { NONE } \\ \text { BIAS/\&CO } & 0.00 \\ \text { EMISSIV } & 0.00\end{array}$


Control Block Configuration - FURN21I

PAGE 14

Nov $061996 \quad 08: 43$ AM

Block Number: 49

Block Type: CMPR

\begin{tabular}{|c|c|c|c|c|}
\hline & & (connected & to) & \\
\hline$\frac{\text { Input Label }}{x}$ & $\frac{\text { Block }}{48}$ & $\frac{\text { Type }}{\text { UAI }}$ & $\frac{\text { output }}{\text { oUT }}$ & Tagname \\
\hline$\hat{y}$ & & & & NC1050 \\
\hline
\end{tabular}

Block Number: 50

Block Type: WDIG

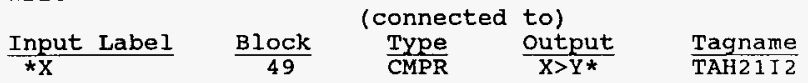

Configuration Parameter Value

ADDRESS 307

Block Number: 51

Block Type: UAI

\begin{tabular}{lll} 
Configuration & Parameter & Value \\
\cline { 1 - 2 } START ADR & 32 \\
INPUT ADR & 42 \\
I/O TYP & LOCAL \\
INPUT TYP & DIR SENSOR \\
CHAR & K TC \\
CHAR UNIT & DEG C \\
AI HI & 1315.56 \\
AI LO & -17.78 \\
SCAN RATE & 0.8 SEC \\
SW FILTER & 2 SEC \\
BURNOUT & ENABLED \\
CRD FSAFE & UPSCALE \\
PROCESSNG & NONE \\
BIAS/\&CO & 0.00 \\
EMISSIV & 0.00
\end{tabular}

Block Number: 60

Block Type: WDIG

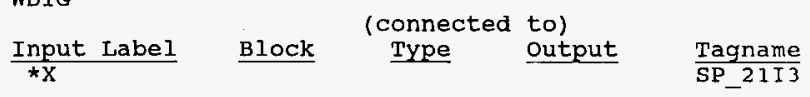

Configuration Parameter $\frac{\text { Value }}{308}$
ADDRESS

ADDRESS 
Control Block configuration - FURN21I

PAGE 15

Nov $06199608: 43$ AM

Block Number: 61

Block Type: RDIG

Configuration Parameter $\frac{\text { Value }}{82}$

Block Number: 62

Block Type: RDIG

Configuration Parameter $\frac{\text { Value }}{98}$

Block Number: 63

Block Type: SPP

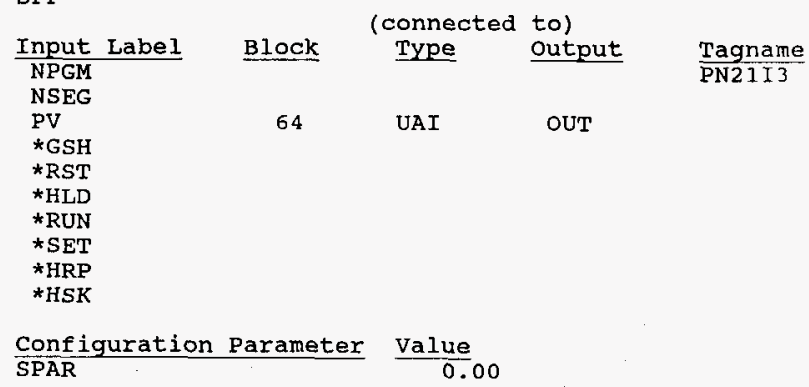


WHC-SD-CP-CSWD-018, Rev. 0

Control Block Configuration - FURN21I

PAGE 16

Nov 06 1996 08:43 AM

Block Number: 64

Block Type: UAI

\begin{tabular}{ll} 
Configuration Parameter & Value \\
\cline { 1 - 2 } START ADR & 32 \\
INPUT ADR & 36 \\
I/O TYP & LOCAL \\
INPUT TYP & DIR SENSOR \\
CHAR & K TC \\
CHAR UNIT & DEG C \\
AT HI & 1315.56 \\
AT IO & -17.78 \\
SCAN RATE & 0.8 SEC \\
SW FILTER & 2 SEC \\
BURNOUT & ENABLED \\
CRD FSAFE & UPSCALE \\
PROCESSNG & NONE \\
BIAS/ 8 CO & 0.00 \\
EMISSIV & 0.00
\end{tabular}


Block Number: 65

Block Type: PID1

\begin{tabular}{|c|c|c|c|c|}
\hline & & (connected & to) & \\
\hline Input Label & Block & Type & output & Tagname \\
\hline PV & 64 & UAI & OUT & \\
\hline RSP & & SPP & SP & 113 \\
\hline
\end{tabular}

Configuration Parameter Value

$\begin{array}{lc}\text { GAIN } & \\ \text { RATE MIN } & 0.00 \\ \text { RSET MIN } & 1.00 \\ \text { CONT ALG } & \text { PID A } \\ \text { PV HI } & 1200.00 \\ \text { PV LO } & 0.00 \\ \text { SP TRACK } & \text { TRACK RSP } \\ \text { RSP UNITS } & \text { ENGR } \\ \text { INIT MODE } & \text { AUTO RSP } \\ \text { PWR MODE } & \text { LAST MODE } \\ \text { PWR OUT } & \text { FAILSAFE } \\ \text { SP HILIM } & 1000.00 \\ \text { SP LOLIM } & 0.00 \\ \text { ACTION } & \text { REVERSE } \\ \text { OUTHILIM } & 100.00 \\ \text { OUTLOLIM } & 0.00 \\ \text { FAILSAFE } & 0.00 \\ \text { A1S1 VAL } & 0.00 \\ \text { A1S2 VAL } & 0.00 \\ \text { A2S1 VAL } & 20.00 \\ \text { A2S2 VAL } & 20.00 \\ \text { A1S1TYPE } & \text { NONE } \\ \text { A1S2TYPE } & \text { NONE } \\ \text { A2S1TYPE } & \text { DEV LO } \\ \text { A2S2TYPE } & \text { DEV HI } \\ \text { AL HYST } & 0.10 \\ \text { TXFR ALG } & \text { DISABLED } \\ \text { REG ADDR } & 4096 \\ \text { AT TYPE } & \text { SP ONLY } \\ \text { SP STEP } & 100.00 \\ \text { AT STATE } & \text { ENABLE } \\ & \\ \text { ST } & \end{array}$


Block Number: 66

Block Type: AO

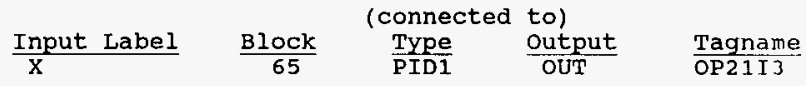

\begin{tabular}{lr} 
Configuration Parameter & \multicolumn{1}{c}{ Value } \\
ADDRESS & 100.00 \\
X HI & 0.00
\end{tabular}

Block Number: 67

Block Type: WDIG

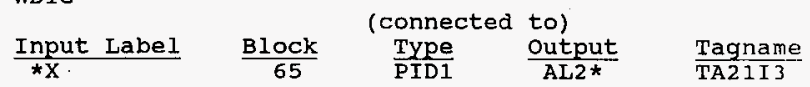

$\frac{\text { Configuration Parameter }}{\text { ADDRESS }} \frac{\text { value }}{309}$

Block Number: 68

Block Type: UAI

\begin{tabular}{|c|c|}
\hline Configuration Parameter & Value \\
\hline START ADR & 32 \\
\hline INPUT ADR & 37 \\
\hline $\mathrm{I} / \mathrm{O}$ TYP & LOCAL \\
\hline INPUT TYP & DIR SENSOR \\
\hline CHAR & $\mathrm{K} \mathrm{TC}$ \\
\hline CHAR UNIT & DEG $C$ \\
\hline AI HI & 1315.56 \\
\hline AI LO & -17.78 \\
\hline SCAN RATE & $0.8 \mathrm{SEC}$ \\
\hline SW FILTER & 2 SEC \\
\hline BURNOUT & ENABLED \\
\hline CRD FSAFE & UPSCALE \\
\hline PROCESSNG & NONE \\
\hline BIAS/\&CO & 0.00 \\
\hline EMISSIV & 0.00 \\
\hline
\end{tabular}


Control Block Configuration - FURN21I

PAGE 19

Nov $061996 \quad 08: 43 \quad A M$

Block Number: 69

Block Type: CMPR

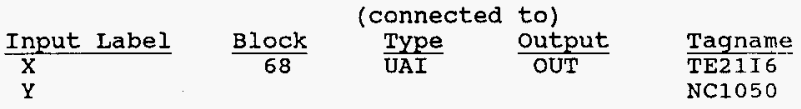

Block Number: 70

Block Type: WDIG

$\frac{\text { Input Label }}{\star X} \quad \frac{B l o c k}{69} \quad \begin{gathered}\begin{array}{c}\text { (connected to) } \\ \text { Type }\end{array} \\ \text { CMPR }\end{gathered} \frac{\text { Output }}{\mathrm{X}>\mathrm{Y} *} \quad \frac{\text { Tagname }}{\text { TAH21I3 }}$

Configuration Parameter Value ADDRESS

310

Block Number: 71

Block Type: UAI

\begin{tabular}{ll} 
Configuration Parameter & Value \\
\cline { 1 - 2 } START ADR & 32 \\
INPUT ADR & 43 \\
I/O TYP & LOCAL \\
INPUT TYP & DIR SENSOR \\
CHAR & K TC \\
CHAR UNIT & DEG C \\
AI HI & 1315.56 \\
AI LO & -17.78 \\
SCAN RATE & 0.8 SEC \\
SW FILTER & 2 SEC \\
BURNOUT & ENABLED \\
CRD FSAFE & UPSCALE \\
PROCESSNG & NONE \\
BIAS/\&CO & 0.00 \\
EMISSIV & 0.00
\end{tabular}


Point Configuration - FURN21I PAGE 1

$$
\text { Nov } 06199608: 43 \mathrm{AM}
$$

Loop Points

TC_21I1 Description:

Security Areas:

Scan Time (seconds):

Block /Num:

Decimal Place:

Engineering Units:

Engineering Units: 1 DEG ${ }^{C}$

Engineering Limit Low: 0.000

PC Spvsr Alarm Enabled: No

TC_21I2 Description:

Security Areas:

Scan Time (seconds):

Block/Num:

Decimal Place:

Engineering Units:

Engineering Limit High:

Engineering Limit Low:

PC Spvsr Alarm Enabled:

TC_21I3 Description:

Security Areas:

Scan Time (seconds):

Block /Num:

Decimal Place:

Engineering Units:

Engineering limit High:

Engineering Limit Low: 0.000

PC Spvsr Alarm Enabled: No
TEMPERATURE CONTROLLER FUR-21I-1

none, none, none

$5: 0$

PID1 25

1

TEMPERATURE CONTROLLER FUR-2II-2

none, none, none

$1: 0$

PID1 45

1.

DEG C

0.000

TEMPERATURE CONTROLLER FUR-21I-3

none, none, none

1:0

PID1 65

1

DEG C

Set Point Program Points

$\begin{array}{lll}\text { TP_21I1 } & \text { Description: } & \text { TEMPERATURE PROGRAMS FOR FUR-21I-1 } \\ & \text { Security Areas: } & \text { none, none, none } \\ \text { Scan Time (seconds): } & 2: 0 \\ \text { Block /Num: } & \text { SPP } 23\end{array}$


Set Point Program points

TP 2112 Description:

Security Areas:

Scan Time (seconds):

Block /Num:

TP_21I3 Description:

Security Areas:

Scan Time (seconds):

Block/Num:
TEMPERATURE PROGRAMS FUR-21I-2

none, none, none

$2: 0$

SPP 43

TEMPERATURE PROGRAMS FUR-21I-3

none, none, none

$2: 0$

SPP 63

Analog Signal Points

OP21I1 Description:

Security Areas:

CONTROLLER OUPPUT FUR-21I-1

Scan Time (seconds): none, none, none

Block/Num:

Output:

Decimal Place:

5:0

PID1 25

OUT

Engineering Units:

Engineering Limit High: 100.0

Engineering Limit Low: 0.000

Communication Address: 25:10

PC Spvsr Alarm Enabled: No 
Analog Signal Points

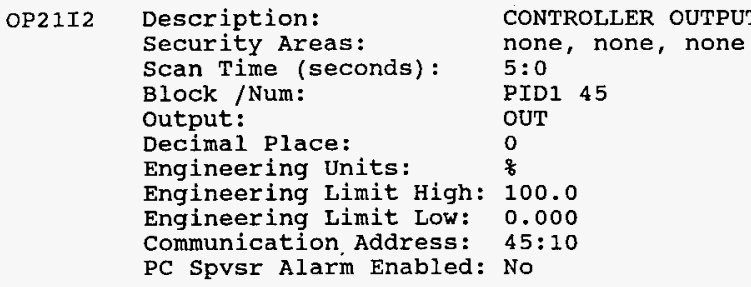


Point Configuration - FURN21I Nov $06 \quad 1996 \quad 08: 43$ AM

Analog Signal points

SP21I1

\section{Description: \\ Security Areas:}

Scan Time (seconds):

Block /Num:

output:

Decimal Place:

Engineering Units:

Engineering

Engineering limit

Communication Address: $23: 8$

PC Spvsr Alarm Enabled: No

SP21I2

Description:

Security Areas:

Scan Time (seconds):

Block / Num:

Output:

Decimal Place:

Engineering Units:

Engineering Limit High: 1200

Engineering Limit Low: 0.000

Communication Address: 43:8

PC Spvsr Alarm Enabled: No
SET POINT FUR-21I-2

none, none, none

$5: 0$

SPP 43

SP

0

DEG C

No 
Analog Signal points

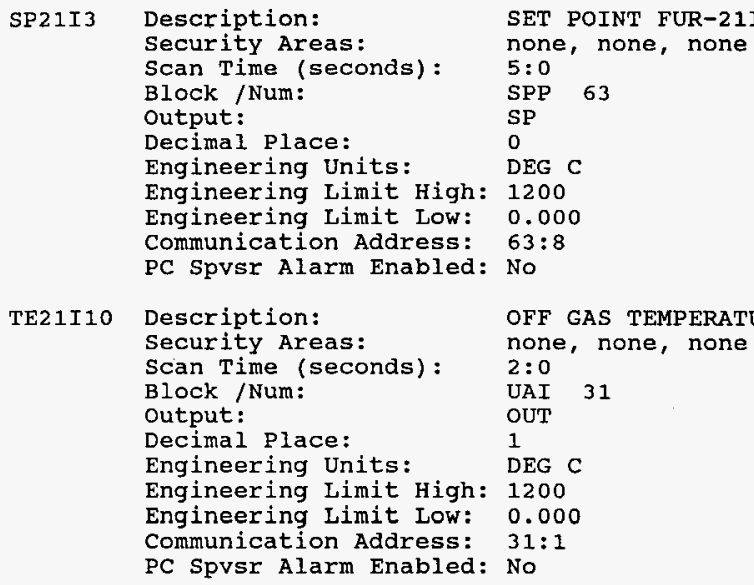


Point Configuration - FURN21I

Nov $06 \quad 1996 \quad 08: 43$ AM

Analog Signal Points

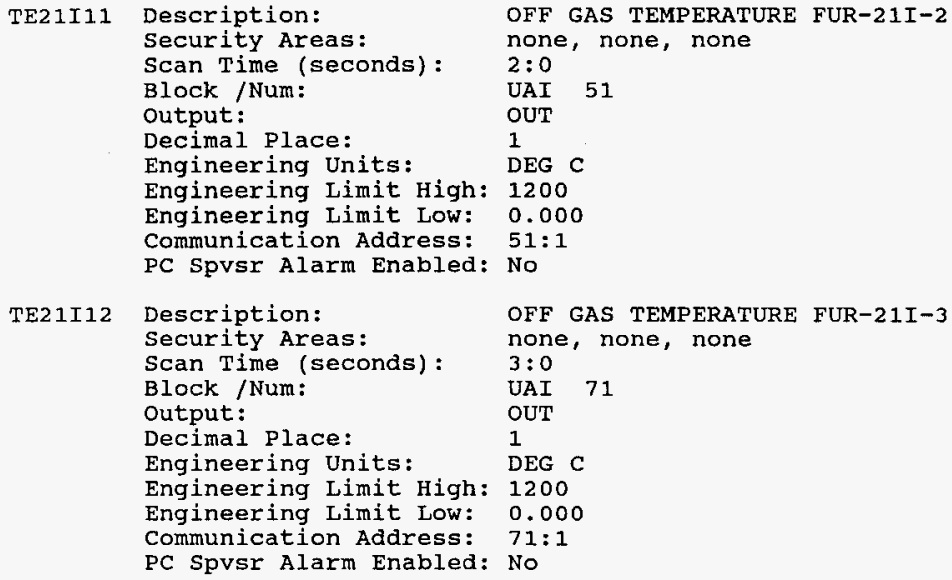


WHC-SD-CP-CSWD-018, Rev. 0

Point configuration - FURN21I

Nov $061996 \quad 08: 43$ AM

I'AGE 7

Analog Signal Points

TE21I2 Description:

Security Areas:

Scan Time (seconds):

High Temperature FUR-21I-1

Block /Num:

Output :

none, none, none

Decimal Place:

$2: 0$

UAI 28

Engineering Units:

OUT

Engineering Limit High: 1200

Engineering Limit Low: 0.000

Communication Address: 28:1

PC Spvsr Alarm Enabled: No

TE21I4

Description :

High Temperature FUR-21I-2

Security Areas:

Scan Time (seconds):

Block /Num:

none, none, none

Output:

Decimal Place:

$2: 0$

UAI 48

OUT

Engineering Units: DEG C

Engineering Limit High: 1200

Engineering Limit Low: 0.000

Communication Address: $48: 1$

PC Spvsr Alarm Enabled: No 


\section{Analog Signal points}

TE21I 6

Description:

Security Areas :

Scan Time (seconds):

Block /Num:

Output:

Decimal Place:

Engineering Units:

Engineering Limit High: 1200

Engineering Limit Low:

Communication Address:

PC Spvsr Alarm Enabled:

TE21I7

\section{Description:}

Security Areas:

Scan Time (seconds):

Block /Num:

Output:

Decimal Place:

Engineering Units:

Engineering Limit $\mathrm{High}$ : 100.0

Engineering Limit Low: 0.000

Communication Address: $7: 1$

PC Spvsr Alarm Enabled: No

2:0

OUT

1

DEG C
High Temperature FUR-21I-3

none, none, none

$2: 0$

UAI 68

OUT

DEG $\mathrm{C}$

0.000

$68: 1$

GLOVEBOX HA-21I TE

none, none, none

UAI 7

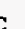


Point Configuration - FURN21I IAGE 9 Nov $06 \quad 1996 \quad 08: 43$ AM

Analog Signal Points

$\begin{array}{lll}\text { TE21I8 } & \text { Description: } & \text { GLOVEBOX HA-21I TE } \\ \text { Security Areas: } & \text { none, none, none } \\ \text { Scan Time (seconds): } & 2: 0 \\ \text { Block /Num: } & \text { UAI } 8 \\ \text { Output: } & \text { OUT } \\ \text { Decimal Place: } & 1 \\ \text { Engineering Units: } & \text { DEG C } \\ \text { Engineering Limit High: } 100.0 \\ \text { Engineering Limit LOW: } 0.000 \\ \text { Communication Address: } 8: 1 \\ \text { PC Spvsr Alarm Enabled: No }\end{array}$

Description:

Security Areas:

Scan Time (seconds):

Block /Num:

Output:

Decimal Place:

Engineering Units:

Engineering Limit $\mathrm{High}$ : 100.0

Engineering Limit Low: 0.000

Communication Address: $9: 1$

PC Spvsr Alarm Enabled: No
GLOVEBOX HA-21I TE none, none, none

2:0

UAI 9

OUT

1

DEG C

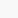

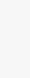


Analog Signal Points

\section{TE21IMAX Description:}

Security Areas:

Scan Time (seconds):

Block /Num:

output:

Decimal Place:

Engineering Units:

Engineering Limit High:

Engineering Limit Low:

Communication Address:

PC Spvsr Alarm Enabled: No

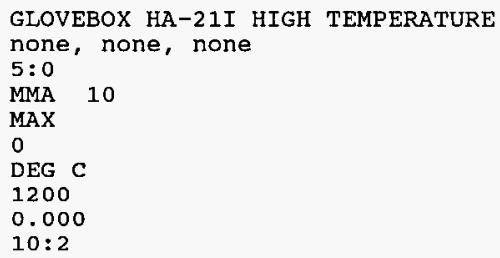

Digital signal Points

FAN1_ON Description:

Security Areas:

Scan Time (seconds):

Block / Num:

Output:

off Label:

On Label:

Communication Address: $4: 1$

OFF-GAS FAN \#1 POWER INDICATION

none, none, none

2:0

RDIG 4

OUT*

OFF

ON

op Panel Alarm Enabled: No

PC Spvsr Alarm Enabled: No

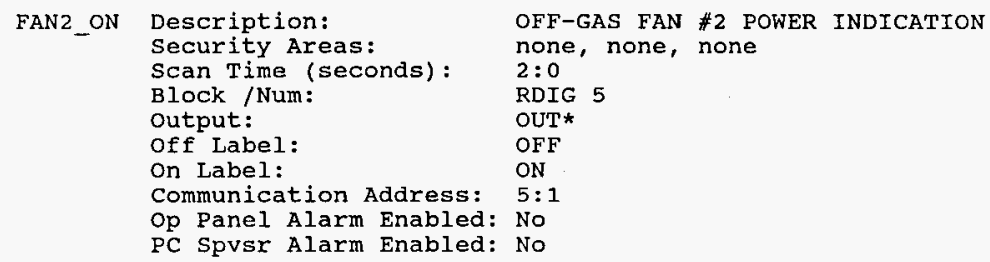


Digital Signal points

FAN_ON Description:

Security Areas:

Scan Time (seconds):

Block /Num:

Output:

off Label:

on Label:

Communication Address:

op Panel Alarm Enabled: No

PC Spvsr Alarm Enabled: No

\section{Description:}

Security Areas:

Scan Time (seconds):

Block /Num:

Output:

Off Label:

On Label:

Communication Address: 21:1

op Panel Alarm Enabled: No

PC Spvsr Alarm Enabled: No

2:0

OUT*

OFF

ON

No

Description:

Security Areas:

Scan Time (seconds):

Block /Num:

output:

Off Label:

on Label:

Communication Address: 41:1

op Panel Alarm Enabled: No

PC Spvsr Alarm Enabled: No

2:0

OUT*

OFF

ON

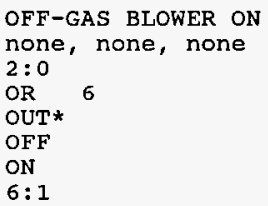

ON

$6: 1$

LIMIT SWITCH FUR-21I-1

none, none, none

RDIG 21

LIMIT SWITCH FUR-2II-2

none, none, none

RDIG 41

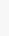


Nov $061996 \quad 08: 43$ AM

Digital Signal points

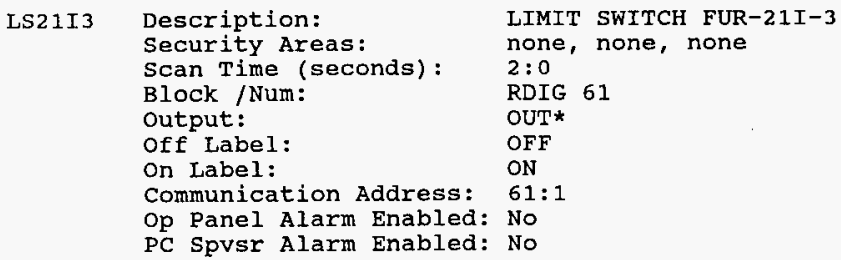

PWR21I2 Description: Security Areas: none, none, none 
Digital Signal Points

PWR21I3 Description:

Security Areas:

Scan Time (seconds):

Block /Num:

output:

off Label:

on Label:

Communication Adaress:

POWER TO FURNACE 21I-3

none, none, none

2: 0

RDIG 62

OUT*

OFF

op Panel Alarm Enabled: No

PC Spvsr Alarm Enabled: No

TA21I1 Description:

DEVIATION ALARM FUR-21I-1

Security Areas:

Scan Time (seconds):

none, none, none

Block /Num:

Output:

2:0

PID1 25

off Label:

AL2 *

On Label:

OFF

Communication Address: 25:9

Op Panel Alarm Enabled: Yes

PC Spvsr Alarm Enabled: Yes

Alarm Area:

Alarm Priority:

all

Low

TA21I2

Description:

Security Areas:

Scan Time (seconds):

Block/Num:

output:

off Label:

on Label:

Communication Address:

op Panel Alarm Enabled:

PC Spvsr Alarm Enabled:

Alarm Area:

Alarm Priority:
DEVIATION ALARM FUR-21I-2

none, none, none

2:0

PID1 45

AL2 *

OFF

ON

$45: 9$

Yes

Yes

all

Low 
Digital Signal points

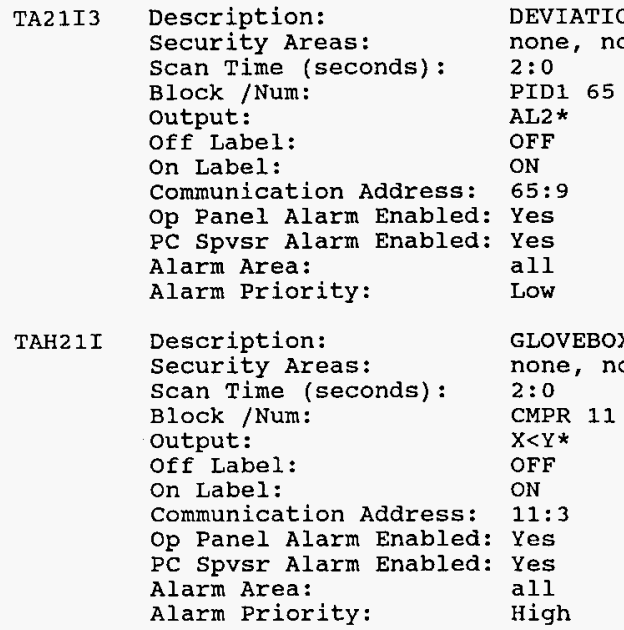


Digital Signal points

$\begin{array}{ll}\text { TAH21I2 } & \text { Description: } \\ \text { Security Areas: } & \text { none, none, none } \\ \text { Scan Time (seconds): } & 2: 0 \\ \text { Block /Num: } & \text { CMPR } 49 \\ \text { Output: } & \text { X>Y* } \\ \text { Off Label: } & \text { OFF } \\ \text { On Label: } & \text { ON } \\ \text { Communication Address: } 49: 1 \\ \text { Op Panel Alarm Enabled: Yes } \\ \text { PC Spvsr Alarm Enabled: Yes } \\ \text { Alarm Area: } & \text { all } \\ \text { Alarm Priority: } & \text { High } \\ & \\ \text { Description: } & \text { FUR-21I-3 HIGH TEMPERATURE } \\ \text { Security Areas: } & \text { none, none, none } \\ \text { Scan Time (seconds): } & 2: 0 \\ \text { Block /Num: } & \text { CMPR } 69 \\ \text { Output: } & \text { X>Y* } \\ \text { Off Label: } & \text { OFF } \\ \text { On Label: } & \text { ON } \\ \text { Communication Address: } 69: 1 \\ \text { Op Panel Alarm Enabled: Yes } \\ \text { PC Spvsr Alarm Enabled: Yes } \\ \text { Alarm Area: } & \text { all } \\ \text { Alarm Priority: } & \text { High }\end{array}$


Point Configuration - FURN21I

AGE 16 Nov $06 \quad 1996 \quad 08: 43 \quad \mathrm{AM}$

Analog Operator Points

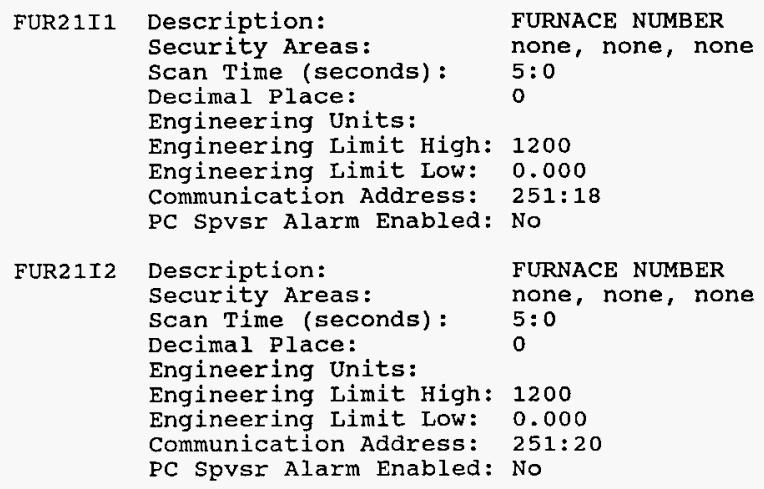

Communication Address: 251:19

FURNACE NUMBER none, none, none $5: 0$

0 

Nov $061996 \quad 08: 43$ AM

Analog Operator Points

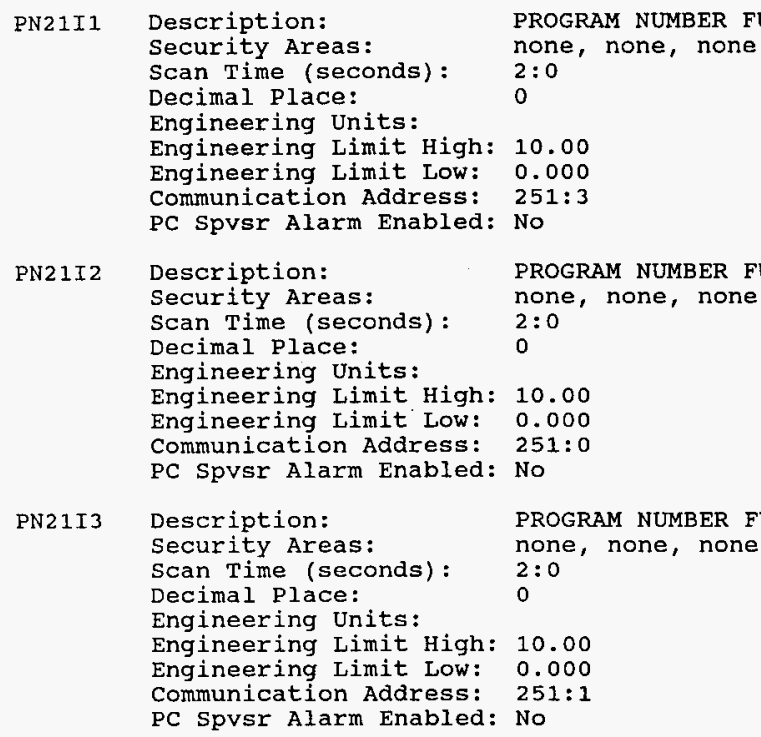



NOV $06 \quad 1996 \quad 08: 43$ AM

Digital operator Points

HS_EMERG Description: Security Areas: Scan Time (seconds): off Label:

on Label:

Communication Address: 251:2

op Panel Alarm Enabled: No

PC Spvsr Alarm Enabled: No

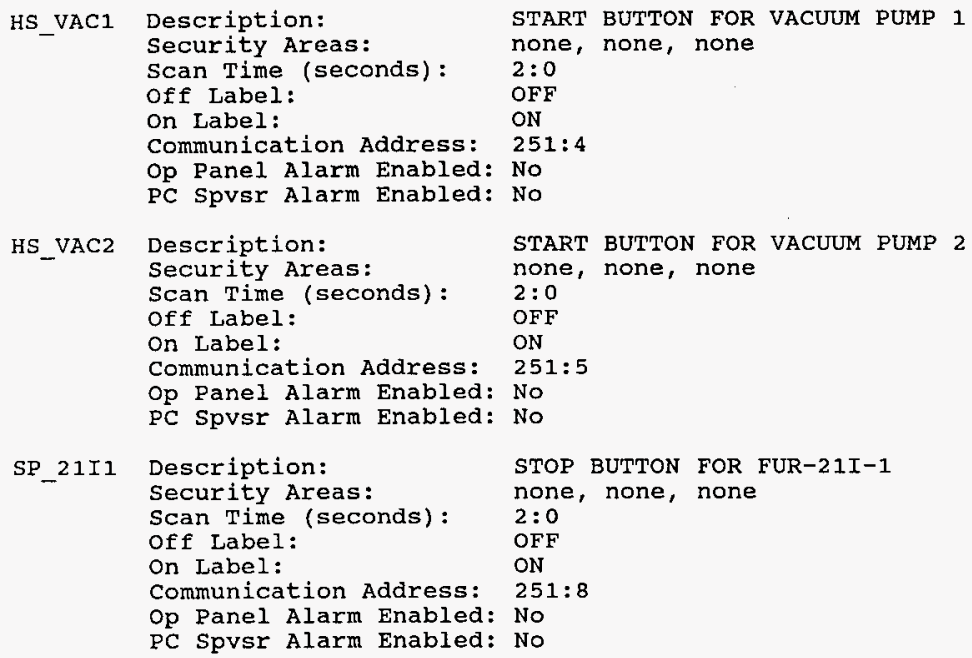


WHC-SD-CP-CSWD-018, Rev. 0

Point configuration - FURN21I

AGE 19

Nov $06 \quad 1996 \quad 08: 43 \quad \mathrm{AM}$

Digital operator Points

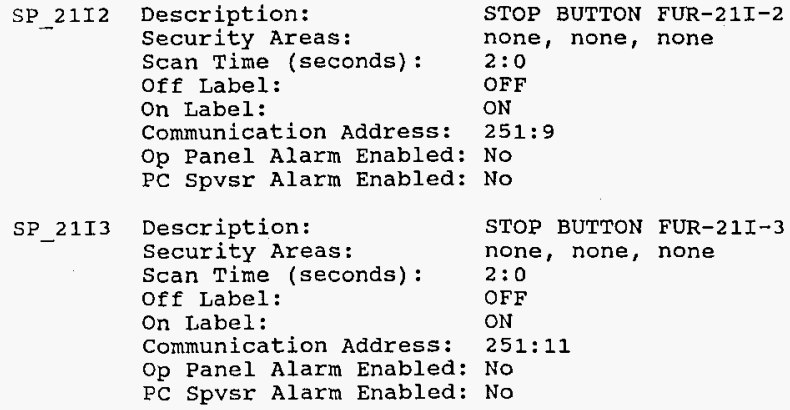


WHC-SD-CP-CSWD-018, Rev. 0

\section{APPENDIX B \\ PCS LADDER LOGIC}


Line \# 1

Line \# Cross-Ref of addr 96

$\star 1$

!HA21I HA21I STRT/STP FUR-21I-1 FUR-21I-1 FUR21II

IEMERGENCY HTAS BUTTON DEVIATION HI TEMP DOOR LS

ISTOP FUR-21I-1 ALARM ALARM

! HSEMERG TAH21I SP 21II TA2III TAH21I1 LS21I2

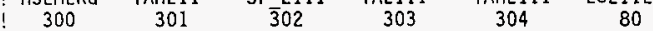

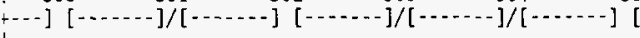

$(0)(0)(0)(0)(0), 10)$

Line $\# \quad 2$

Line \# Cross-Ref of addr 97

*2

IHA21I HA21I STRT/STP FUR-21I-2 FUR-21I-2 FUR21I2

!EMERGENCY HTAS BUTTON DEVIATION HI TEMP DOOR LS

ISTOP BUTTON DEVIATION HI TEMP
FUR-21I-2 ALARM ALARM

1 HSEMERG TAH21I SP 2112 TA2II2 TAH2II2 L\$21I4

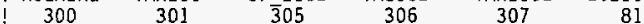

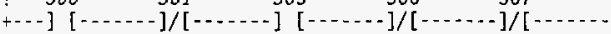

$\vdots(0)(0)(0)(0) 10)(0)$

! Line \# 3

Line \# Cross-Ref of addr 98

*3

!EMERGENCY HTAS BUTTON DEVIATION HI TEMP DOOR LS

ISTOP

! HSEMERG TAH21I SP 21I3 TA21I3 TAH2113 LS21I6

START

FUR2 III

CONTACTOR

PWR21II!

$96 !$

$\begin{array}{llllll}1 & 300 & 301 & 308 & 309 & 310\end{array}$

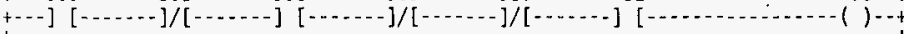

$!(0)(0)(0)(0)(0) 10)$

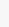

\begin{tabular}{|c|c|c|}
\hline 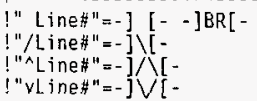 & $\begin{array}{l}\text { "tline\#"=TMR/CTR } \\
\text { "fLine\#"=- [FP]- } \\
\text { "FLine\#"=-(FP)- } \\
\text { "eL ine\#"= error addr for: }\end{array}$ & $\begin{array}{l}\text { "mL ine } \# "=M A T R I X \text { REF. ADDR } \\
\text { "MLine\#"=MATRIX OUTPUT ADOR } \\
\text { "bL ine" }=-[B 2]-\quad-<B 2>- \\
\text { "SL ine" }=-(S 2)-\quad-<I 2>- \text { SEQNCR! }\end{array}$ \\
\hline
\end{tabular}


WHC-SD-CP-CSWD-018, Rev. 0

\begin{tabular}{|c|c|}
\hline $\begin{array}{l}\text { "zLine\#" }=-[Z]- \\
\text { "*Line\#" }=-(\text { (Z)- }-(R)- \\
-(L)--(U)--(B W)-\end{array}$ & $\begin{array}{l}-[+]--[-]--[*]--[/]-" s L \text { ine\#"=SEQNCR STEP \# } \\
-[\text { [BCD]- }-[B I N]--[\text { INT]- "pLine\#"=-[PUL] } \\
-[\text { SQRT]-[ABS]- } \\
\text { "PLine\#" }=-(\text { PSH) }-\end{array}$ \\
\hline
\end{tabular}

;20-12 :Mem $S z=2048: P g m ~ S z=21 \quad:$ Lines=3 :Reg $S z=256$ :Forces $=0$

Listing begun> Wed Nov 20 22:04 1996,

Listing ended> Wed Nov 20 22:04 1996. 
WHC-SD-CP-CSWD-018, Rev. 0

\section{APPENDIX C \\ SET POINT PROGRAMS}




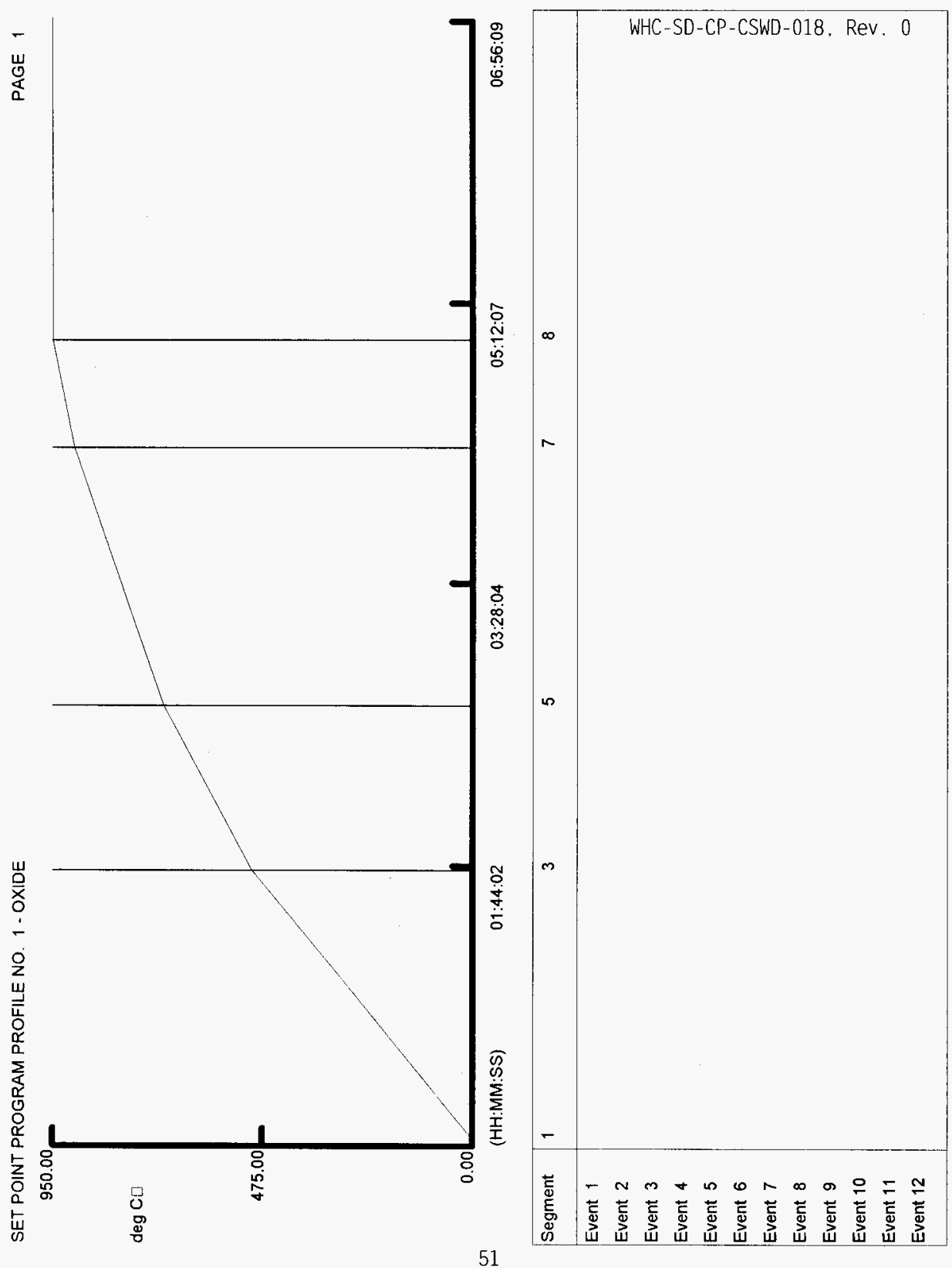




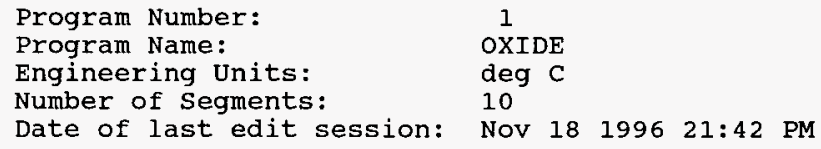

Date of last edit session: Nov 18 1996 21:42 PM

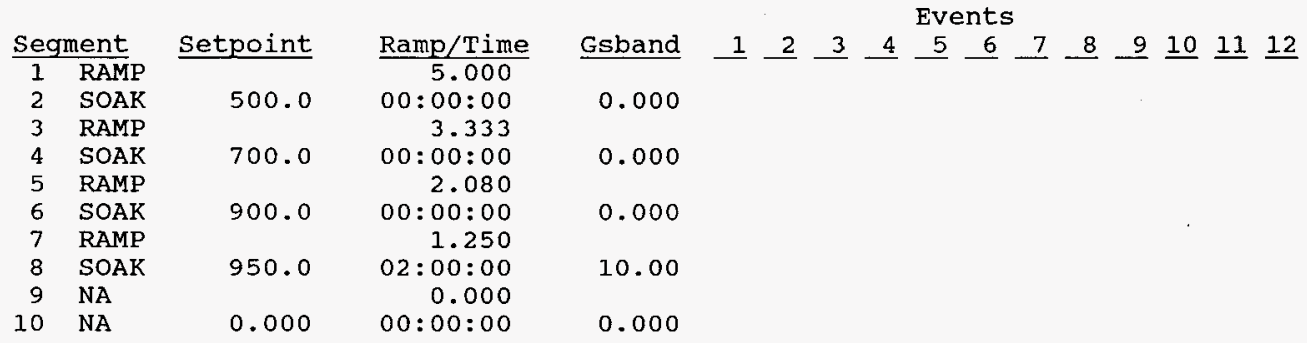




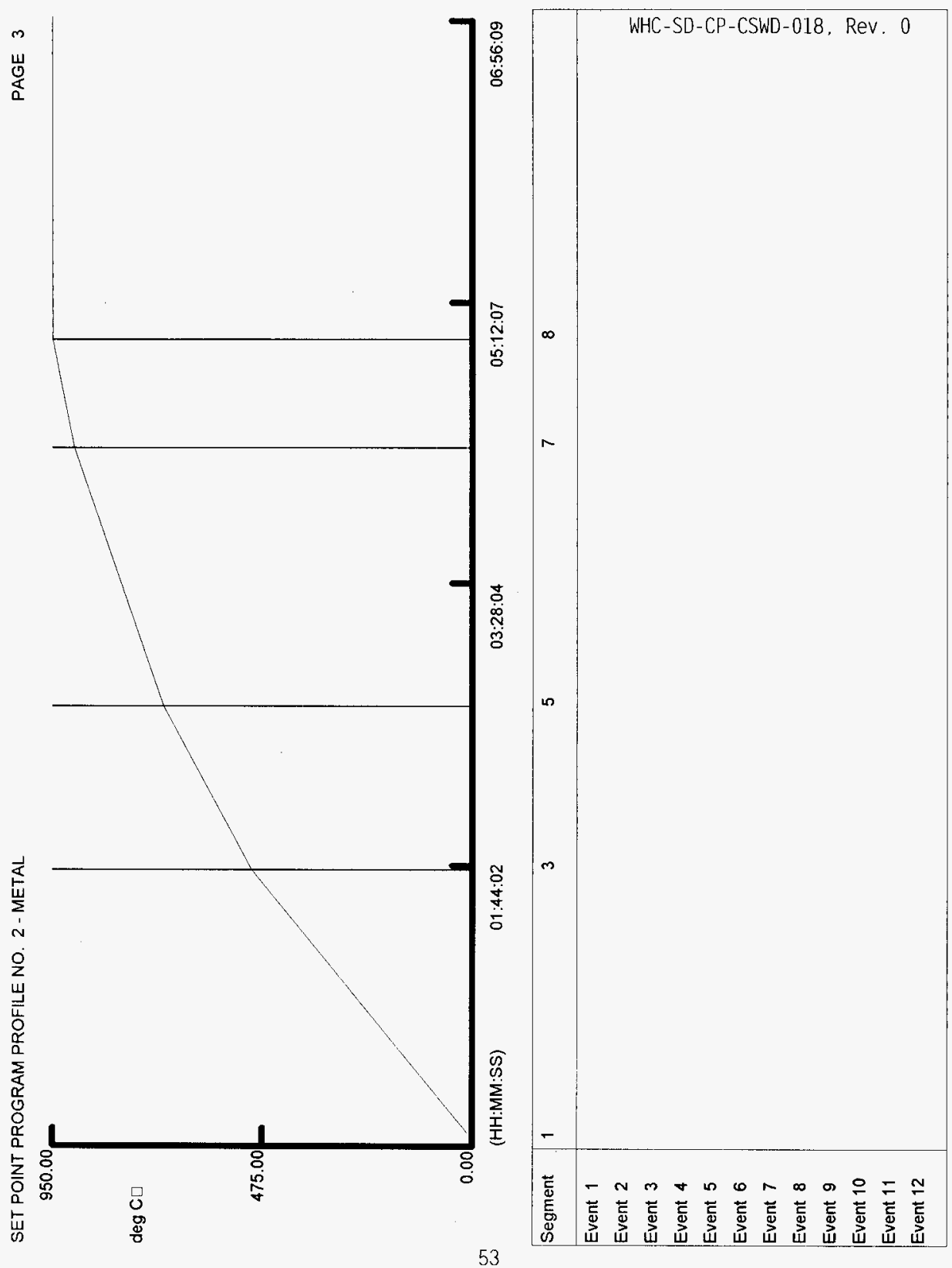


Program Number:

Program Name:

2

Engineering Units:

METAL

Number of Segments:

deg $C$

Date of last edit session:

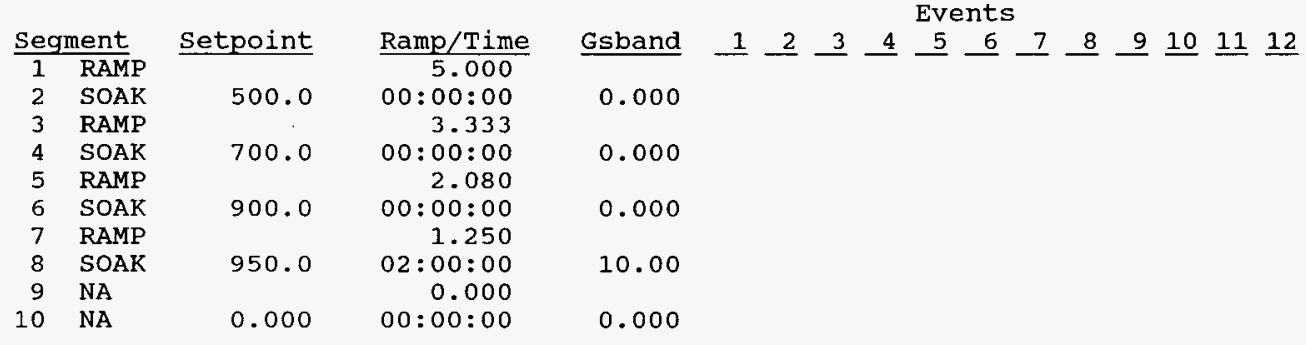




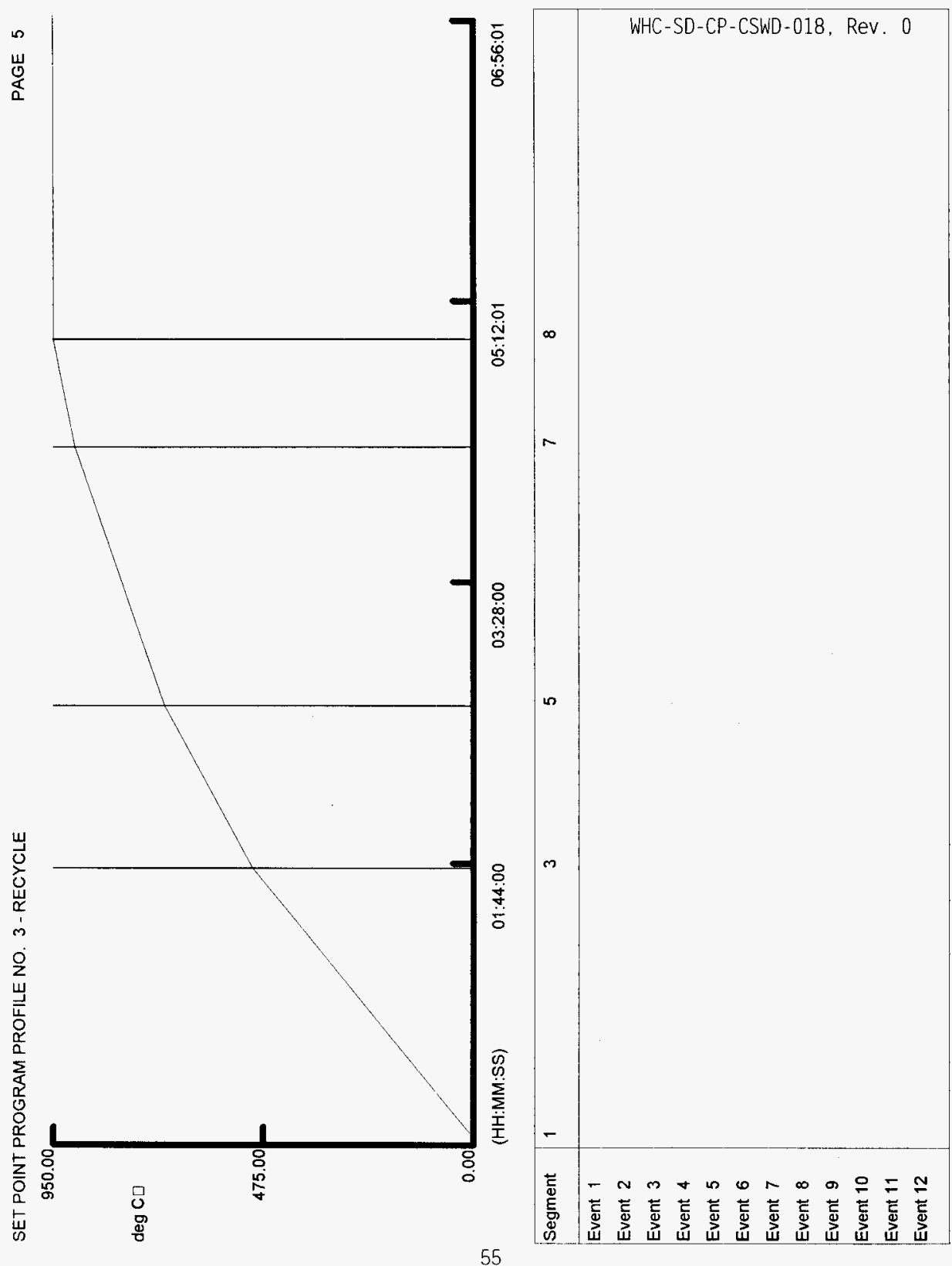


Program Number:

Program Name:

Engineering Units:

Number of Segments:

3

RECYCLE

deg $C$

10

Oct $14 \quad 1996 \quad 08: 42$ AM

Date of last edit session:

Events

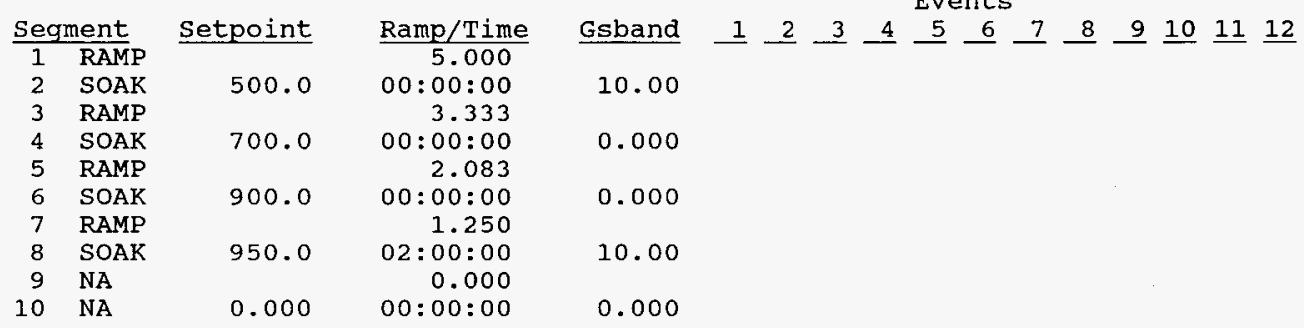


WHC-SD-CP-CSWD-018, Rev. 0

\section{APPENDIX D \\ GRAPHIC DISPLAYS AND REPORTS}




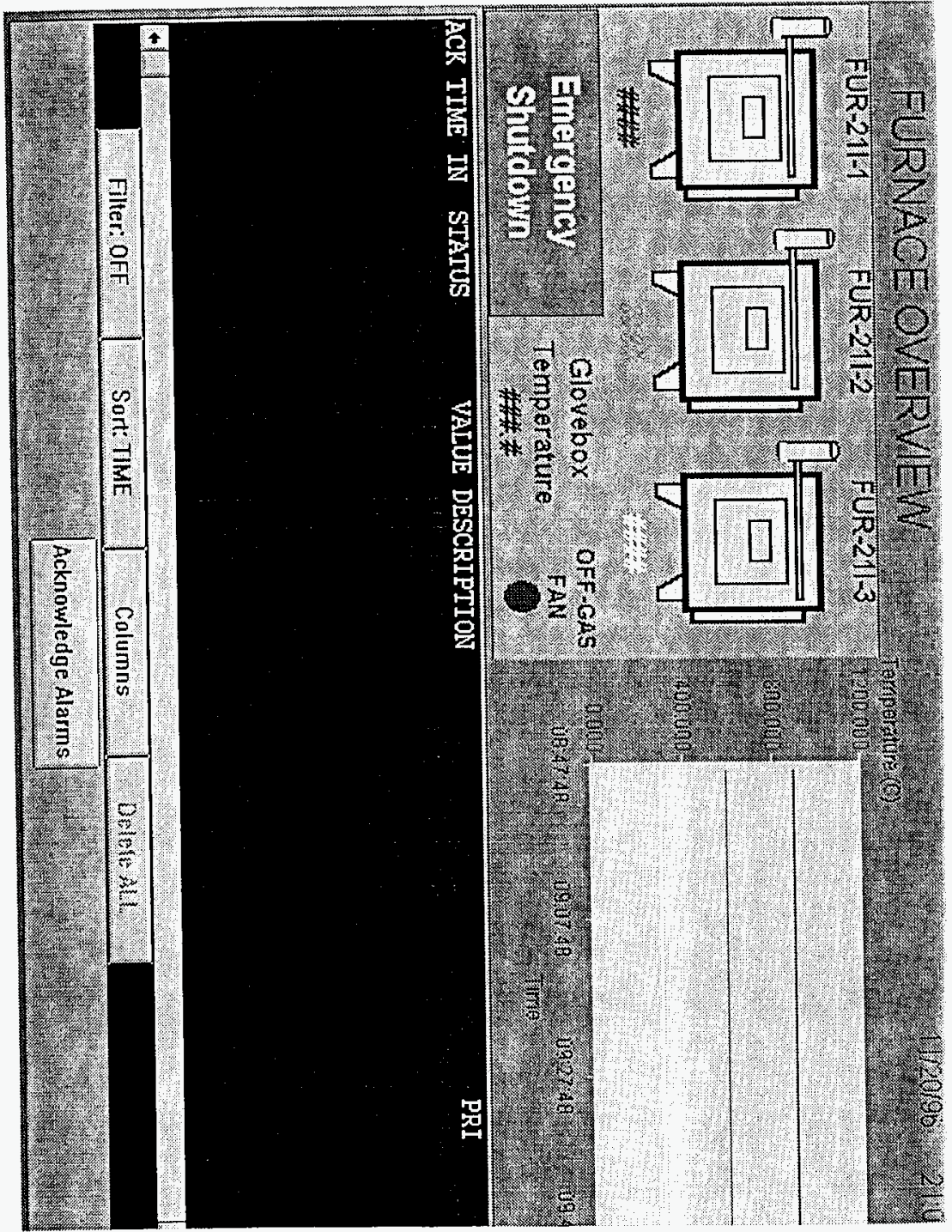


FIX DMACS for Windows Dynamic Properties Report

Report Date: 11/06/96

Report Time: 08:14:02

Report File Name:

ODF Version:

FURNOVER.ODR

$800 f$

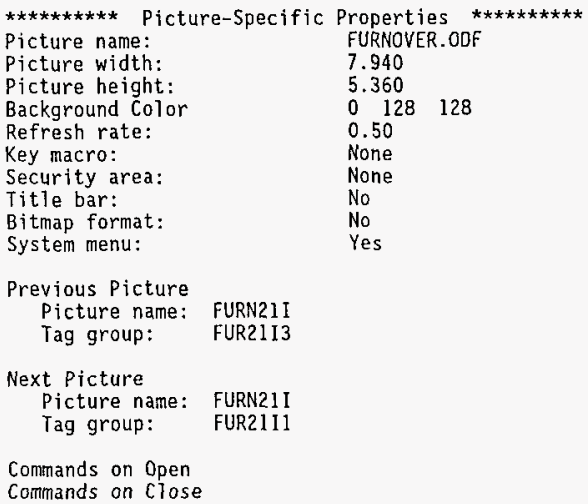


Dynamic Properties Report, FURNOVER. ODR

Object: RECT 9, 360, 4050, 2490

Controllable: 0

Modifiable: 0

Object: TEXT $350,360,1110,560$

Text: FUR-211-1

Object: TEXT $1650,360,2410,560$

Text: FUR-21I-2

Object: TEXT $2940,360,3700,560$

Text: FUR-21I-3

Object: TEXY $540,10,3270,330$

TeXt: FURNACE OVERVIEW

Object: DATALINK $520,1610,890,1810$

Controllable: 0

Modifiable: 0

Tagname: PCS:TC_21I1.F_CV

Visible Background Colors: 255, 255, 255

Object: DATALINK $1840,1610,2210,1810$

Controllable: 0

Modifiable: 0

Tagname: PCS:TC_21I2.F_CV

Visible Background Colors: 255, 255, 255

Object: DATALINK $3140,1610,3510,1810$

Contrallable: 0

Modifiable: 0

Tagname: PCS:TC_2113.F_CV 
Visible Background Colors: 255, 255, 255

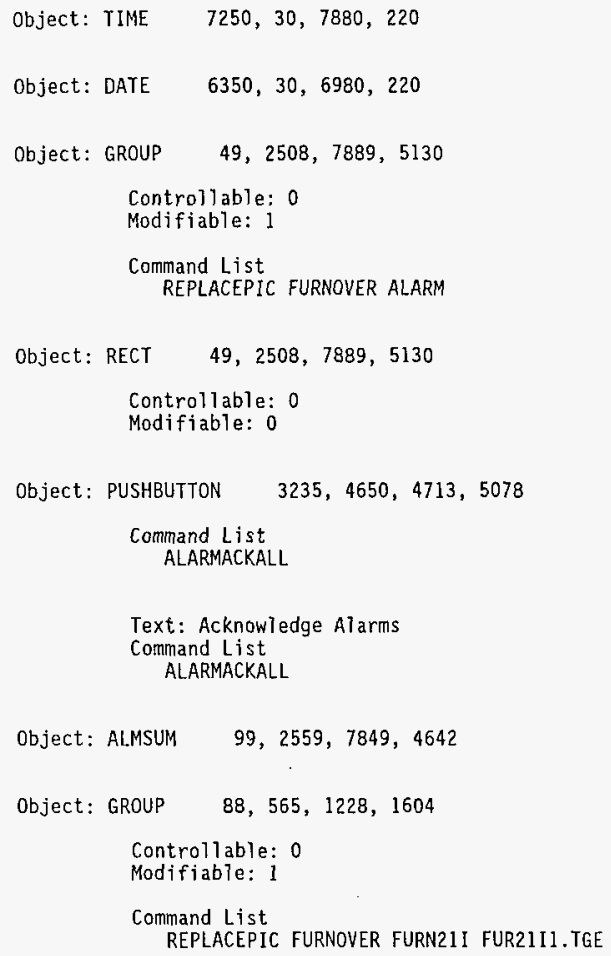


Controliable: 0

Modifiable: 0

Object: PGON $338,1418,533,1604$

Controllable: 0

Modifiable: 0

Object: PGON $900,1418,1095,1604$

Controllable: 0

Modifiable: 0

Object: RECT $284,658,1170,1459$

Controllable: 0

Modifiable: 0

Object: RECT $190,775,1099,845$

Controllable: 0

Modifiable: 0

Object: GROUP $\quad 88,565,243,937$

Controllable: 0

Modifiable: 0

Object: RECT $88,585,243,915$

Controllable: 0

Modifiable: 0

Object: OVAL $90,565,243,654$

Controllable: 0

Modifiable: 0

Object: OVAL $90,850,243,937$

Controllable: 0

Modifiable: 0

Object: RECT 103,854, 228, 904 
Controllable: 0

Modifiable: 0

Object: RECT 524, 940, 904, 1242

Controllable: 0

Modifiable: 0

Object: GROUP $439,852,1003,1279$

Controllable: 0

Modifiable: 0

Vertical Position

Tagname: PCS:LS2IIL.F_CV

Low Limit: 0.00 Minimum offset: -0.25

Hi Limit: 1.00 Maximum offset: 0.00

Allow Input Properties: 0

Object: RECT 439, 852, 1003, 1279

Controllable: 0

Modifiable: 0

Object: RECT $\quad 573,936,861,1092$

Controllable: 0

Modifiable: 0

Object: GROUP $1388,565,2528,1604$

Controllable: 0

Modifiable: 1

Command list

REPLACEPIC FURNOVER FURN21I FUR21I2. TGE

Object: GROUP $1388,565,2528,1604$

Controllable: 0

Modifiable: 0

Object: RECT $2411,760,2528,1406$

Controllable: 0

Modifiable: 0 
Dynamic Properties Report, FURNOVER. ODR

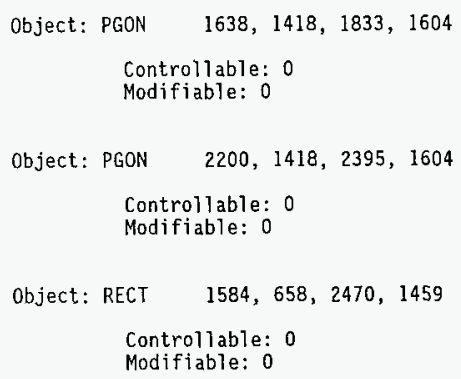

Object: RECT ]824, 940, 2204, 1242 
Controllable: 0

Modifiable: 0

Object: GROUP $1739,852,2303,1279$

Controllable: 0

Modifiable: 0

Vertical Position

Tagname: PCS:LS2112.F_CV

Low Limit: 0.00 Minimum 0ffset: -0.25

Hi Limit: 1.00

Maximum Offset: 0.00

Allow Input Properties: 0

Object: RECT $1739,852,2303,1279$

Controllable: 0

Modifiable: 0

Object: RECT 1873, 936, 2161, 1092

Controllable: 0

Modifiable: 0

Object: GROUP 2688, 564, 3828, 1603

Controllable: 0

Modifiable: 1

Command List

REPLACEPIC FURNOVER FURN21I FUR21I3.TGE

Object: GROUP $2688,564,3828,1603$

Controllable: 0

Modifiable: 0

Object: RECT $3711,759,3828,1405$

Controllable: 0

Modifiable: 0

Object: PGON 2938, 1417, 3133, 1603

Controllable: 0

Modifiable: 0 


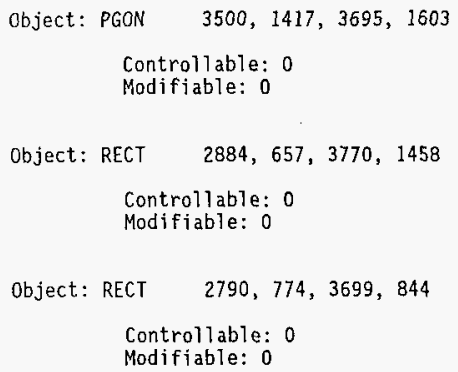

Object: GROUP $3039,851,3603,1278$ 
Dynamic Properties Report, FURNOVER.ODR

page 9

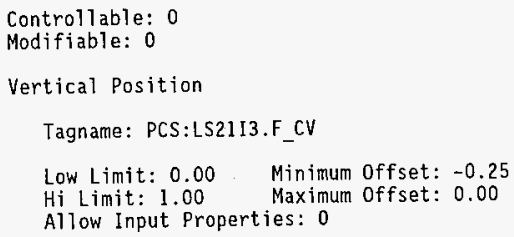




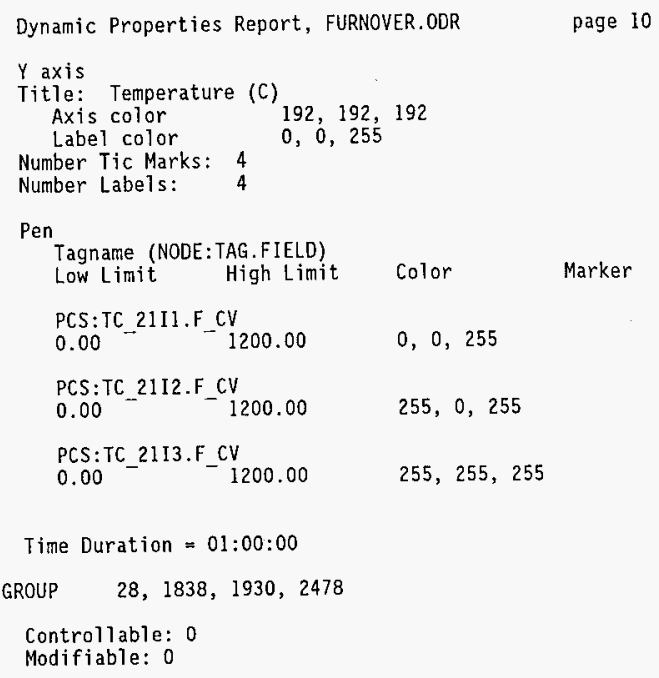

Object: RECT 28, 1838, 1930, 2478

Controllable: 0

Modifiable: 1

Foreground Color by value

Tagname: PCS:HS EMERG.F CV

Blink on new alarm: 0

Value Color Property

$0.00 \quad 128,128,128$ VISIBLE

1

$255,0,0 \quad$ VISIBLE

Command List

TOGGLEDIG PCS:HS EMERG.F_CV

SETVAL PCS:PN21IT.F CV $0^{-}$

SETVAL PCS:TP 2111 C.F 21

SETVAL PCS:PN2]I2.FCV $\overline{0}$

SETVAL PCS:TP $2112{ }^{-} C . F 21$

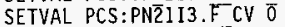

SETVAL PCS:TP_2113_C.F_2 1

Object: TEXT $440,1920,1510,2420$ 
Dynamic Properties Report, FURNOVER.ODR

Shutdown Text: Emergency

Object: GROUP $3350,1890,3990,2430$

Controllable: 0

Modifiable: 1

Command List

REPLACEPIC FURNOVER OFFGAS

Object: TEXT $3350,1890,3990,2210$

FAN Text: OFF-GAS

Object: OVAL $3540,2210,3760,2430$

Controllable: 0

Modifiable: 0

Foreground color by value

Tagname: PCS:FAN_ON.F_CV

Blink on new a la $\bar{r} m: 0^{-}$

$\begin{array}{lll}\text { Value } & \text { Color } & \text { Property } \\ 0 & 255,255,255 & \text { VISIBLE } \\ 1 & 255,0,0 & \text { VISIBLE }\end{array}$

Object: GROUP $2080,1890,3090,2480$

Controllable: 0

Modifiable: 1

Command List

REPLACEPIC FURNOVER GBT21I

Object: TEXT $2080,1890,3090,2290$

Temperaturæext: Glovebox

Object: DATALINK $2360,2280,2780,2480$

Controllable: 0

Modifiable: 0

Tagname: PCS:TE21IMAX.F_CV

Visible Background Colors: 255, 255, 255 page 11 


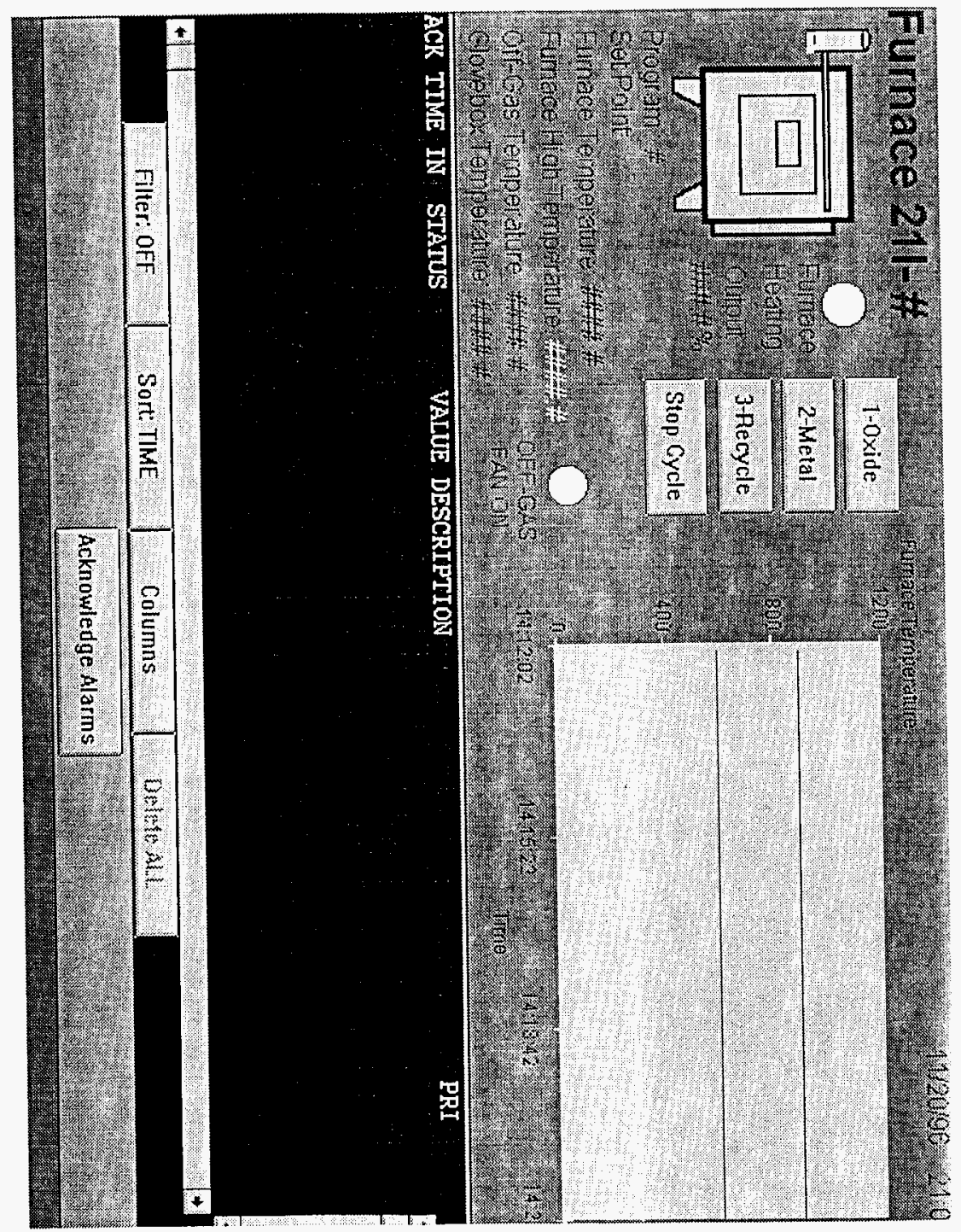


FIX DMACS for Windows

Dynamic Properties Report

Report Date: 11/06/96

Report Time: 08:01:34

Report File Name: FURN211.0DR

ODF Version: $\quad 800 f$

$\begin{array}{ll}* * * * * * * * * \text { Picture-Specific } & \text { Properties } * * * * * * * * * \\ \text { Ficture name: } & 7.940 \\ \text { Picture width: } & 5.360 \\ \text { Picture height: } & 0128 \quad 128 \\ \text { Background Color } & 0.50 \\ \text { Refresh rate: } & \text { None } \\ \text { Key macro: } & \text { None } \\ \text { Security area: } & \text { No } \\ \text { Title bar: } & \text { No } \\ \text { Bitmap format: } & \text { No } \\ \text { System menu: } & \\ & \\ \text { Previous Picture } & \\ \text { Picture name: FURNOVER } & \\ \text { Tag group: } & \text { None } \\ \text { Next Picture } & \\ \text { Picture name: FURNOVER } & \\ \text { Tag group: } & \text { None } \\ \text { Commands on Open } & \\ \text { Commands on Close } & \end{array}$


Object: TEXT $130,1740,820,1930$

Text: Set Point:

Object: TEXT $130,1940,1680,2130$

Text: Furnace Temperature:

Object: TEXT $130,1550,830,1740$

Text: Program :

Object: DATALINK $860,1740,1240,1950$

Controllable: 1

Modifiable: 0

Tagname: ?TEMPSETPOINT

Visible Background Colors: 255, 255, 255

Object: DATALINK 1720, 1930, 2240, 2140

Controllable: 1

Modifiable: 0

Tagname: ?OVENTEMP

Visible Background Colors: 255, 255, 255

Object: TEXT 120,2570,1750, 2760

Text: Glovebox Temperature:

Object: DATALINK $1760,2370,2280,2580$

Controllable: 1

Modifiable: 0

Tagname: ?OFFGASTEMP

Visible Background Colors: $255,255,255$

Object: DATALINK $1780,2580,2300,2790$ 
Dynamic Properties Report, FURN21I.0DR

Controllable: 1

Modifiable: 0

Tagname: ?GBTEMP

Visible Background Colors: 255, 255, 255

Object: TEXT $120,2370,1720,2570$

Text: Off-Gas Temperature:

Object: PENTREND 3318, 142, 7898, 2808

page 3

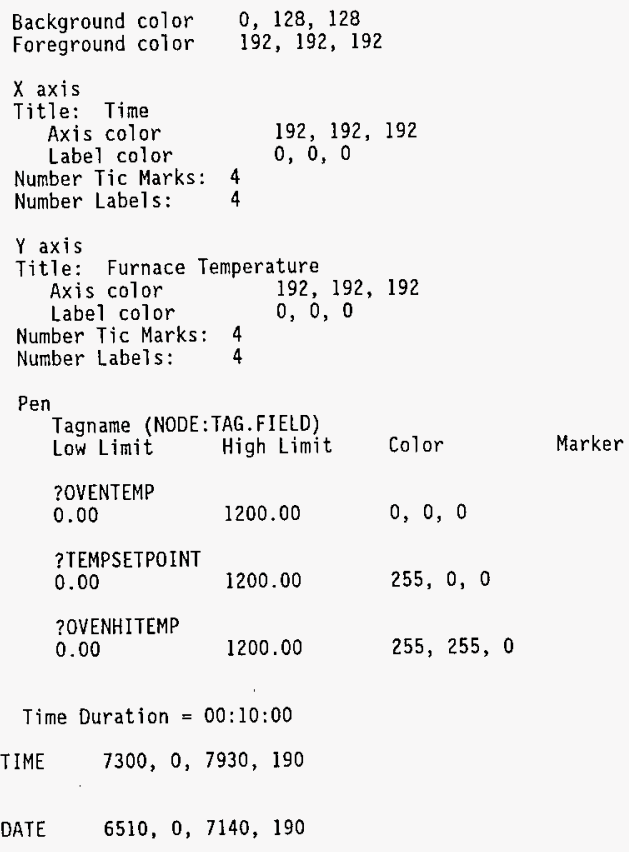

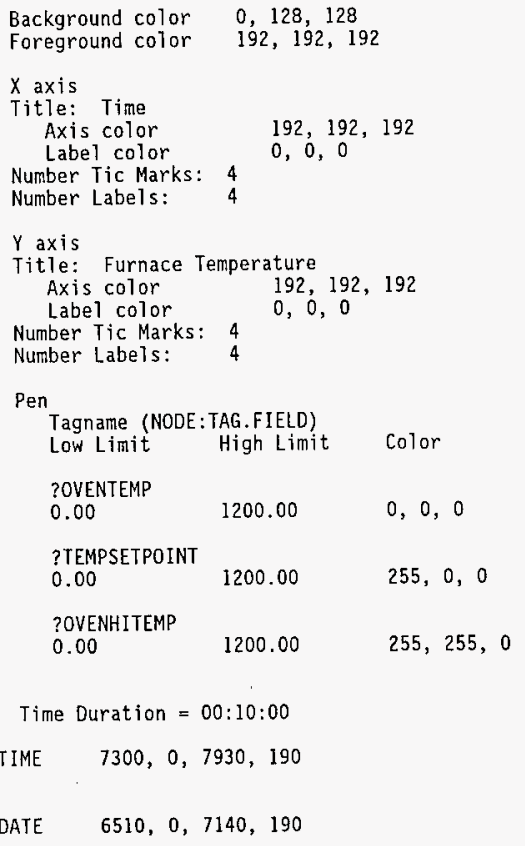

Low Limit High Limit Color Marker 
Dynamic Properties Report, FURN211.ODR

Object: TEXT $130,2150,2040,2340$

Text: Furnace High Temperature:

Object: DATALINK $2060,2150,2580,2360$

Controllable: 0
Modifiable: 0

Tagname: ?OVENHITEMP

Visible Background Colors: 255, 255, 255

Object: DATALINK $870,1550,980,1760$

Controllable: 1

Modifiable: 0

Tagname: ?PROGRAM

Visible Background Colors: 255, 255, 255

Object: RECT $-1,2789,7769,5180$

Controllable: 0

Modifiable: 0

Object: GROUP $\quad 29,2843,7699,5171$

Controllable: 0

Modifiable: 1

Command List

REPLACEPIC FURN2II ALARM

Object: PUSHBUTTON $3183,4749,4655,5171$

Command List

ALARMACKALL

Text: Acknowledge Alarms

Command List

ALARMACKALL

Object: ALMSUM $29,2843,7699,4720$ 
Object: OVAL $1710,430,2010,710$

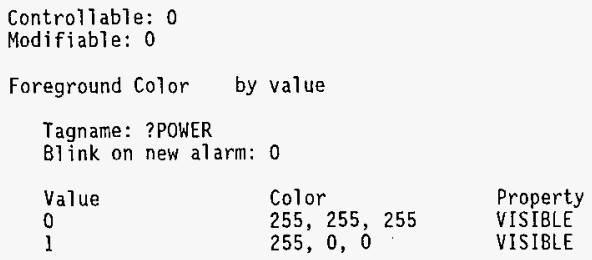

Object: TEXT $1600,680,2180,1060$

Heating Text: Furnace

Object: GROUP $1590,1100,2160,1490$

Controllable: 0

Modifiable: 0

Object: TEXT 2010, 1300,2160, 1490

Text: \%

Object: DATALINK 1590, 1300, 2000, 1490

Controllable: 0

Modifiable: 0

Tagname: ?FURNPOWER

Visible Background Colors: 255, 255, 255

Object: TEXT $1610,1100,2070,1290$

Text: Output

Object: TEXT $10,20,1730,370$

Text: Furnace 211-

Object: DATALINK $1780,20,1960,390$

Controliable: 0

Modifiable: 0 
Dynamic Properties Report, FURN21I.ODR

Tagname: ?FURNNAME

Visible Background Colors: 255, 255, 255

Object: GROUP $2680,2050,3320,2660$

Controllable: 0

Modifiable: 0

Object: OVAL 2825, 2050,3100, 2310

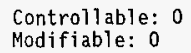

Object: TEXT 2680,2340, 3320, 2660

FAN ON Text: OFF-GAS

Object: GROUP $129,377,1430,1550$

Controllable: 0

Modifiable: I

Command list

REPLACEPIC FURN21 I FURNOVER

Object: RECT $1305,599,1430,1325$

Controllable: 0

Modifiable: 0

Object: PGON 416, 1342, 636, 1550

Controllable: 0

Modifiable: 0

Object: PGON 1060, 1342, 1279, 1550

Controllable: 0

Modifiable: 0 


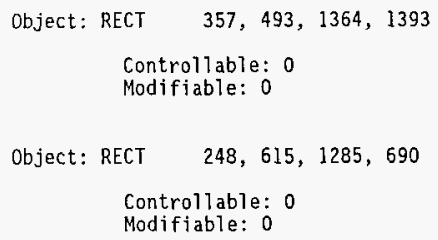


Dynamic Properties Report, FURN21I.0DR

Vertical Position

Tagname: ?LS

Low Limit: 0 Minimum offset: -0

Hi Limit: I Maximum Offset: 0

Al low Input Properties: 0

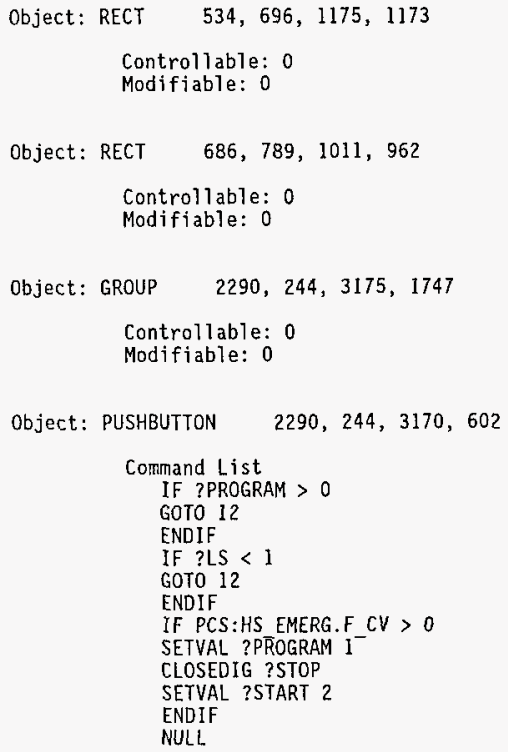

Text: 1-0xide Command List 
Dynamic Properties Report, FURN21I.0DR

page 9

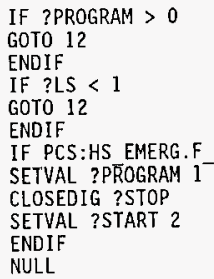

Object: PUSHBUTTON $2291,611,3171,961$

Command List

IF ?PROGRAM >0

GOTO 12

ENDIF

IF $?$ L $S<1$

GOTO 12

ENDIF

IF PCS: HS EMERG.F CV >0

SETVAL ?PREGRAM $2-$

CLOSEDIG ?STOP

SETVAL ?START 2

ENDIF

NULL

\section{Text: 2-Meta]}

Command List

IF ?PROGRAM >0

GOTO 12

ENDIF

IF ?LS $<1$

GOTO 12

ENDIF

IF PCS:HS EMERG.F_CV $>0$

SETVAL ?PREOGRAM $2^{-}$

CLOSEDIG ?STOP

SETVAL ?START 2

ENDIF

NULL

Object: PUSHBUTTON $2294,968,3174,1331$

Command List 
Dynamic Properties Report, FURN21I.ODR

IF ?PROGRAM >0

GOTO 12

ENDIF

IF $?$ LS $<1$

GOTO 12

ENDIF

IF PCS:HS EMERG.F_CV $>0$

SETVAL ?PROGRAM $3^{-}$

CLOSEDIG ?STOP

SETVAL ?START 2

ENDIF

NULL

Text: 3-Recycle

Command List

IF ?PROGRAM >0

GOTO 12

ENDIF

IF ? LS $<1$

GOTO 12

ENDIF

IF PCS:HS EMERG.F CV >0

SETVAL ?PRTOGRAM 3

CLOSEDIG ?STOP

SETVAL ?START 2

ENDIF

NULL.

Object: PUSHBUTTON 2293, 1348, 3175, 1747

Command List

SETVAL ?PROGRAM 0

OPENDIG ?STOP

SETVAL ?START 1

Text: Stop Cycle

Command List

SETVAL ?PROGRAM 0

OPENDIG ?STOP

SETVAL ?START 1 


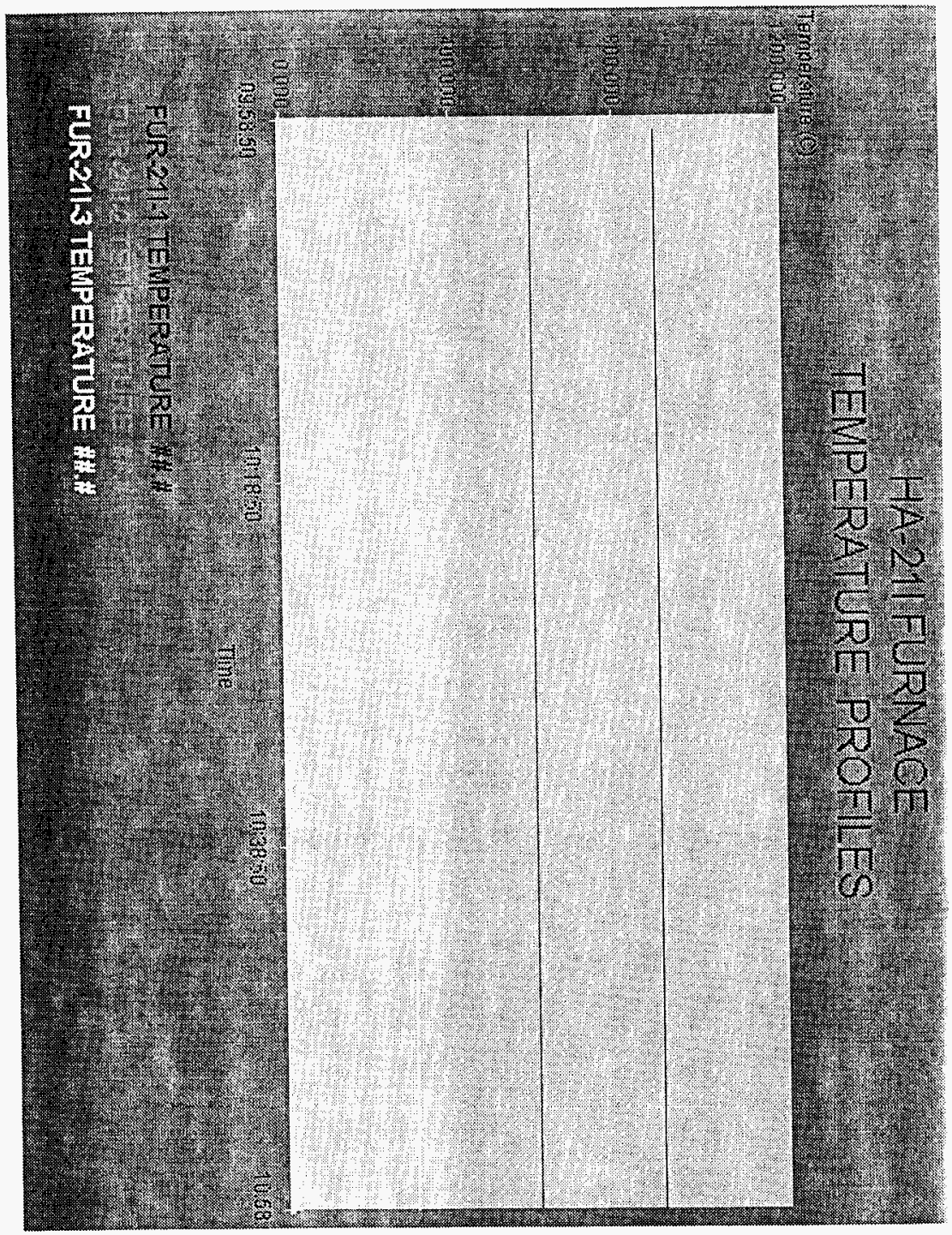


WHC-SD-CP-CSWD-018, Rev. 0

FIX DMACS for Windows

Dynamic Properties Report

Report Date: 11/06/96

Report Time: 08:15:24

Report File Name: TEMPGRAF.OOR

ODF Version: $\quad 800 \mathrm{f}$

$\star \star \star * * * * * * *$ Picture-Specific Properties

Picture name:

Picture width:

TEMPGRAF. ODF

Picture height:

7.940

Background Color

5.360

Refresh rate:

$\begin{array}{lll}0 & 128 & 128\end{array}$

key macro:

Security area:

Title bar:

0.50

None

None

Bitmap format:

System menu:

No

No

Previous Picture

Picture name: None

Tag group: None

Next Picture

Picture name: None

Tag group: None

Commands on Open

Commands on close 
Dynamic Properties Report, TEMPGRAF.ODR

Object: TEXT 2220, 60, 5580,700

TEMPERATURE $X$ RROFILES HA-21I FURNACE

Object: GROUP $\quad 40,670,7800,4350$

Controllable: 0

Modifiable: 1

Command List

REPLACEPIC TEMPGRAF FURNOVER

Object: RECT $141,703,7436,4306$

\author{
Controllable: 0 \\ Modifiable: 1 \\ Command List \\ REPLACEPIC TEMPGRAF FURNOVER
}

Object: PENTREND $\quad 40,670,7800,4350$

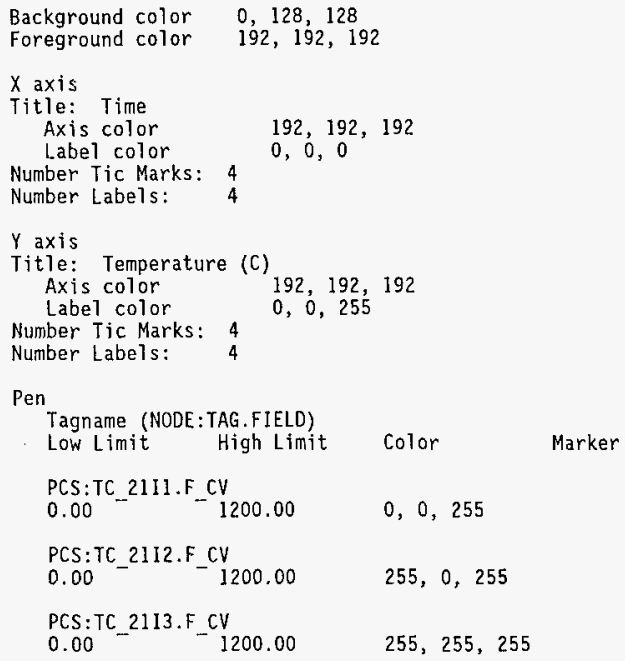


Dynamic Properties Report, TEMPGRAF.ODR

page 3

Time Duration $=01: 00: 00$

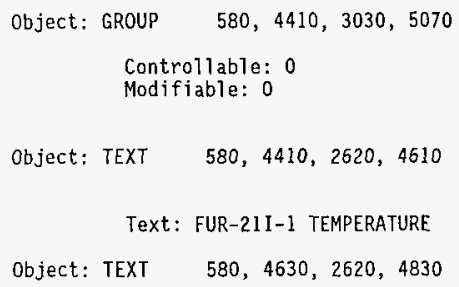

Text: FUR-21I-2 TEMPERATURE

Object: TEXT $580,4850,2620,5050$

Text: FUR-21I-3 TEMPERATURE

Object: DATALINK $2700,4410,3030,4630$

Controllable: 0

Modifiable: 0

Tagname: PCS:TC_2111.F_CV

Visible Background Colors: 255, 255, 255

Object: DATALINK $2700,4630,3030,4850$

Controllable: 0

Modifiable: 0

Tagname: PCS:TC_21I1.F_CV

Visible Background Colors: 255, 255, 255

Object: DATALINK $2700,4850,3030,5070$

Controllable: 0

Modifiable: 0

Tagname: PCS:TC_2111.F CV

Visible Background Colors: 255, 255, 255 


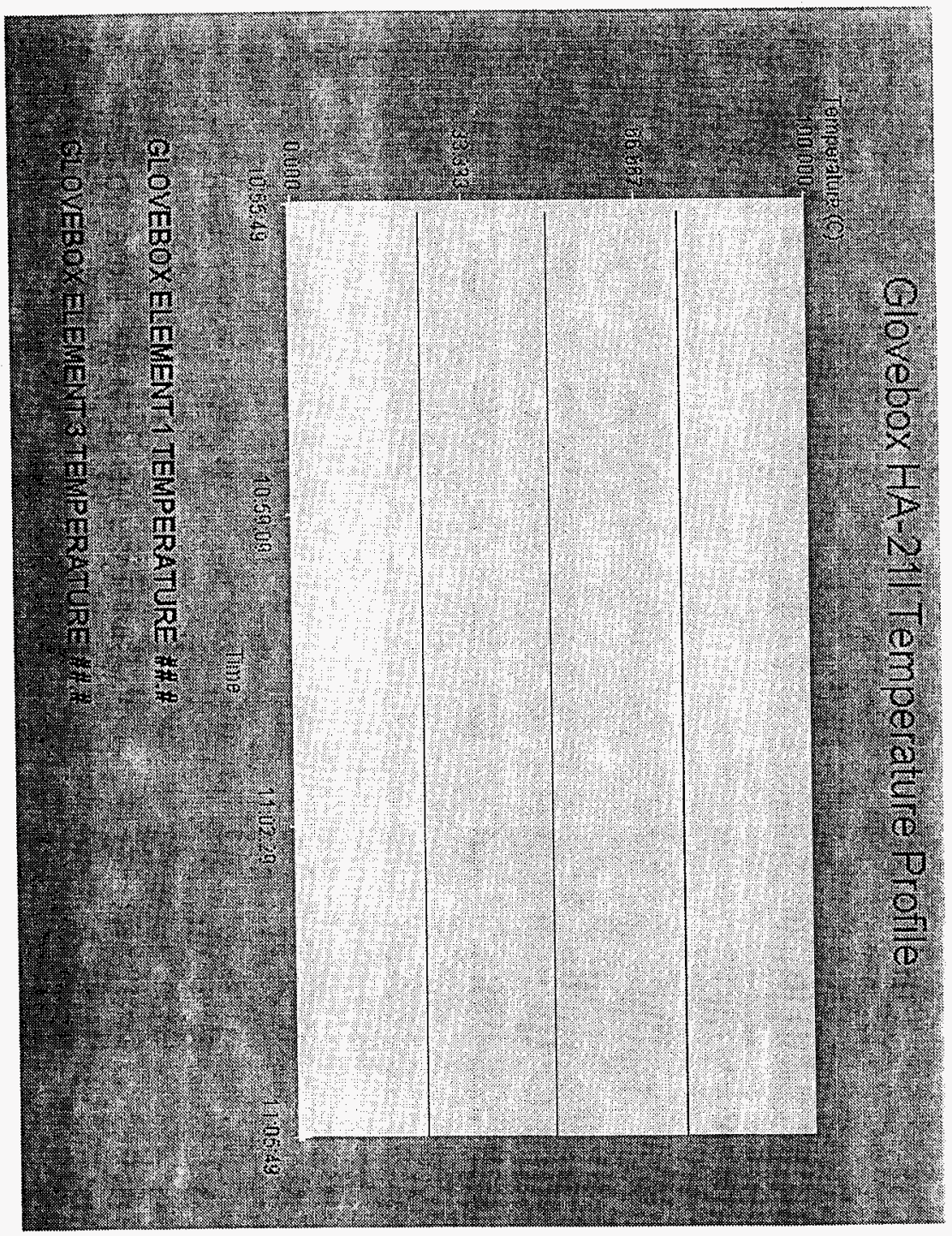


WHC-SD-CP-CSWD-018, Rev. 0

FIX DMACS for Windows

Dynamic Properties Report

Report Date: 11/06/96

Report Time: 08:14:35

Report File Name: GBT211.00R

ODF Version: $800 f$

$\begin{array}{ll}* * * * * * * * * \text { Picture-Specific Properties } \\ \text { Gicture name: } & 7.940 \\ \text { Picture width: } & 5.360 \\ \text { Picture height: } & 0128 \text { 128 } \\ \text { Background Color } & 0.50 \\ \text { Refresh rate: } & \text { None } \\ \text { Key macro: } & \text { None } \\ \text { Security area: } & \text { No } \\ \text { Title bar: } & \text { No } \\ \text { Bitmap format: } & \text { No } \\ \text { System menu: } & \\ \text { Previous Picture } & \\ \text { Picture name: None } & \\ \text { Tag group: } & \text { None } \\ \text { Next Picture } & \\ \text { Picture name: None } & \\ \text { Tag group: } & \text { None } \\ \text { Commands on Open } & \\ \text { Commands on Close } & \end{array}$


Dynamic Properties Report, GBT211.00R . page 2

Object: TEXT $1680,50,5980,370$

Text: Glovebox HA-21I Temperature Profile

Object: GROUP $780,4230,4320,4930$

Controllable: 0

Modifiable: 0

Object: TEXT $\quad 780,4230,3930,4430$

Text: GLOVEBOX ELEMENT I TEMPERATURE

Object: TEXT $780,4470,3930,4670$

Text: GLOVEBOX ELEMENT 2 TEMPERATURE

Object: TEXT $780,4720,3930,4920$

Text: GLOVEBOX ELEMENT 3 TEMPERATURE

Object: DATALINK $3990,4230,4320,4450$

Controllable: 0

Modifiable: 0

Tagname: PCS:TE2117.F_CV

Visible Background Colors: $255,255,255$

Object: DATALINK $3990,4470,4320,4690$

Controllable: 0

Modifiable: 0

Tagname: PCS:TE21I8.F CV

Visible Background Colors: 255, 255, 255

Object: DATALINK $3990,4710,4320,4930$

Controliable: 0

Modifiable: 0

Tagname: PCS:TE21I9.F_CV 
Dynamic Properties Report, GBT211.0DR

page 3

Visible Background Colors: 255, 255, 255

Object: GROUP $\quad 549,489,7319,4140$

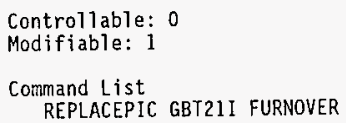

Controllable: 0

Modifiable: 1

Command List

REPLACEPIC GBT21I FURNOVER

Object: RECT $636,579,7131,4068$

Controllable: 0

Modifiable: 0

Object: PENTREND $549,489,7319,4140$

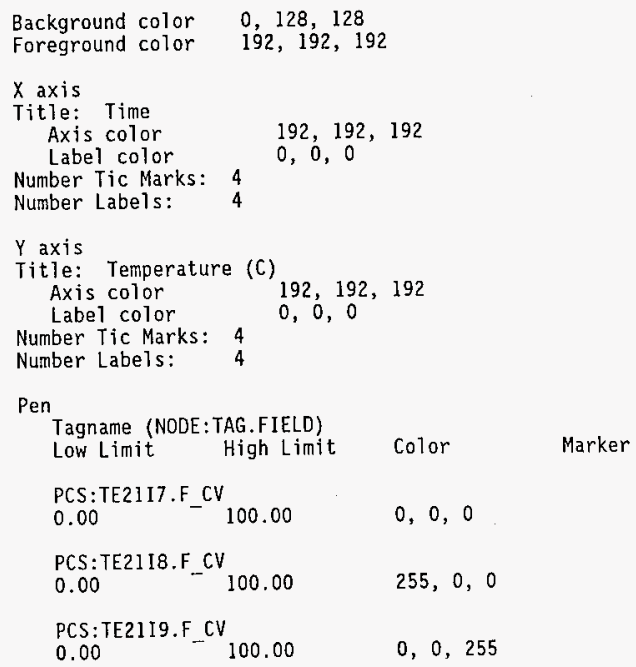

Time Duration $=00: 10: 00$ 
WHC-SD-CP-CSWD-018. Rev. 0

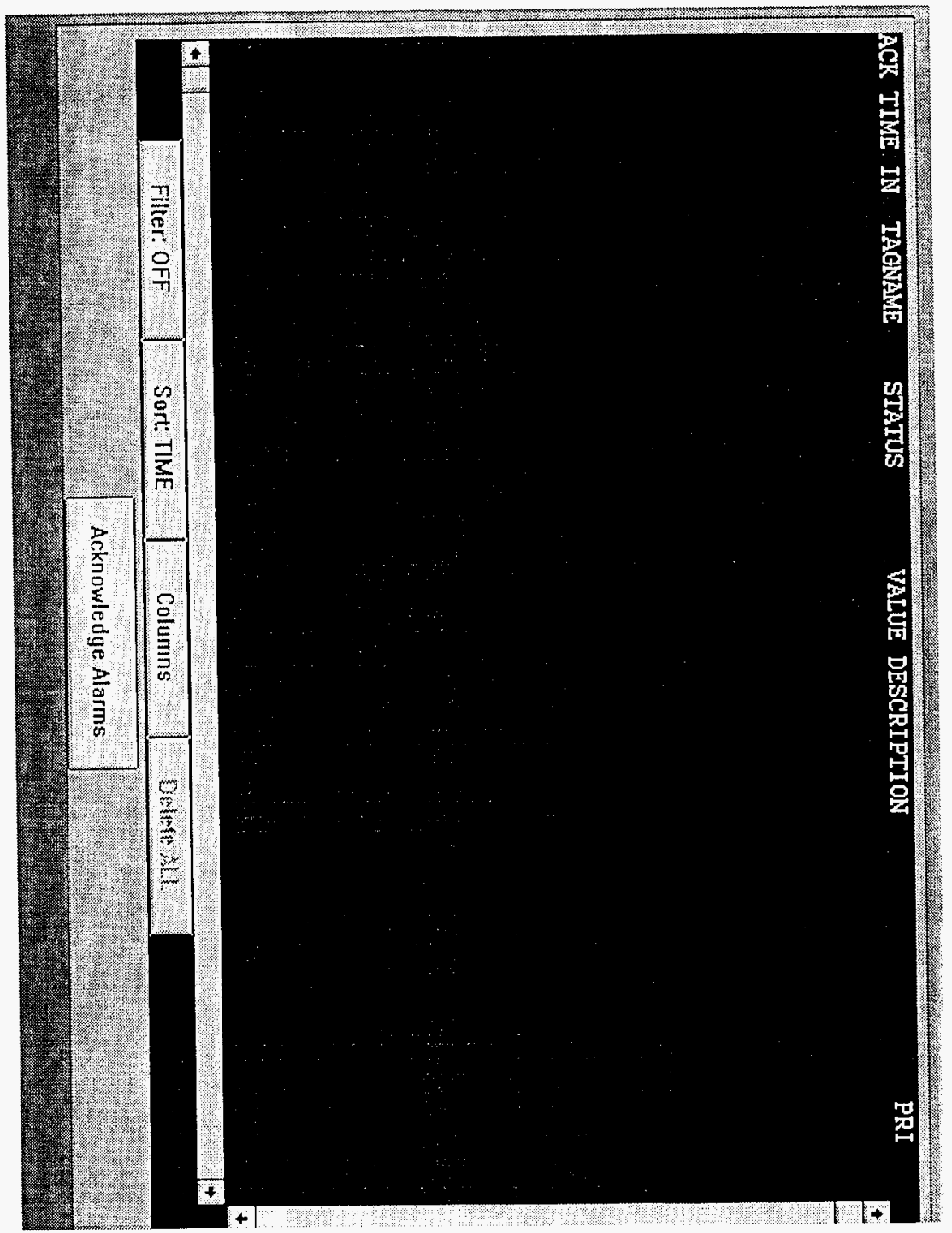


WHC-SD-CP-CSWD-018, Rev. 0

FIX DMACS for Windows

Dynamic Properties Report

Report Date: 11/06/96

Report Time: 08:14:20

Report File Name: ALARM.ODR

ODF Version: $800 \mathrm{f}$

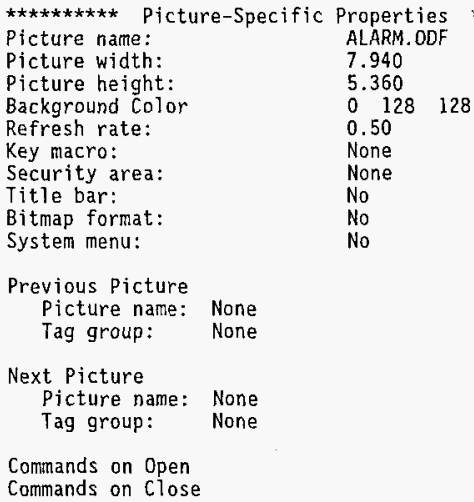


Dynamic Properties Report, ALARM.ODR

Object: GROUP $60,79,7770,5070$

Controllable: 0

ModifiabTe: I

Command List

REPLACEPIC ALARM FURNOVER

Object: PUSHBUTTON $2997,4613,4750,5070$

Command List

ALARMACKALL

Text: Acknowledge Alarms

Command list

ALARMACKALL

Object: RECT $60,79,7770,5058$

Controllable: 0

Modifiable: 0

Object: ALMSUM $\quad 183,169,7633,4579$ 


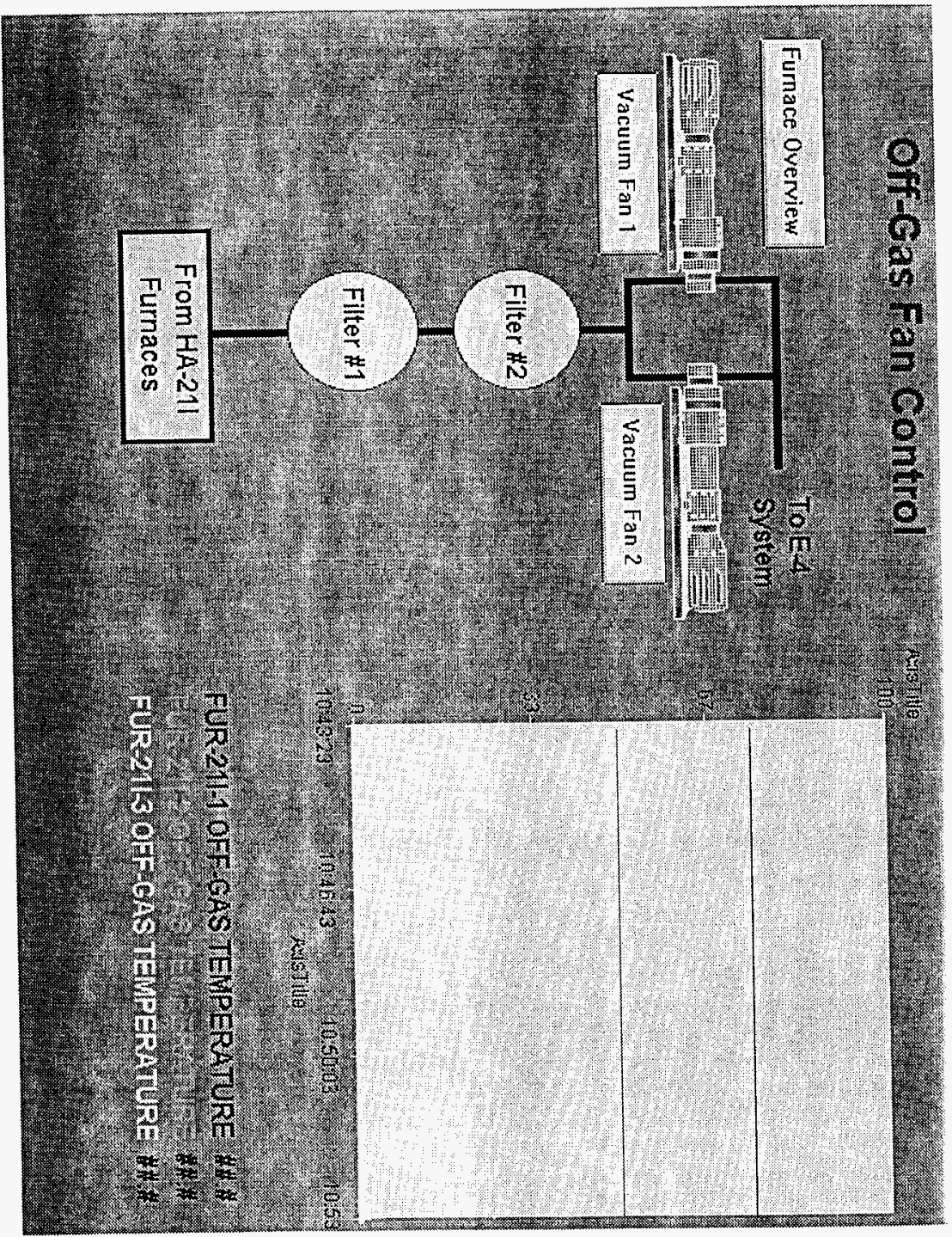


WHC-SD-CP-CSWD-018, Rev. 0

FIX DMACS for Windows

Dynamic Properties Report

Report Date: 11/06/96

Report Time: 08:13:45

Report File Name: OFFGAS.ODR

ODF Version: $\quad 800 \mathrm{f}$

$\begin{array}{ll}* * * * * * * * * \text { Picture-Specific Properties } \\ \text { Picture name: } & \text { OFFGAS.ODF } \\ \text { Picture width: } & 7.940 \\ \text { Picture height: } & 5.360 \\ \text { Background Color } & 0128 \quad 128 \\ \text { Refresh rate: } & 0.50 \\ \text { Key macro: } & \text { None } \\ \text { Security area: } & \text { None } \\ \text { Title bar: } & \text { Yes } \\ \text { Bitmap format: } & \text { No } \\ \text { System menu: } & \text { No } \\ & \\ \text { Previous Picture } & \\ \text { Picture name: None } & \\ \text { Tag group: } & \text { None } \\ \text { Next Picture } & \\ \text { Picture name: None } & \\ \text { Tag group: } & \text { None } \\ \text { Commands on Open } & \\ \text { Commands on Close } & \end{array}$


Dynamic Properties Report, OFFGAS.OOR

page 2

Object: PENTREND $3800,100,7840,3960$

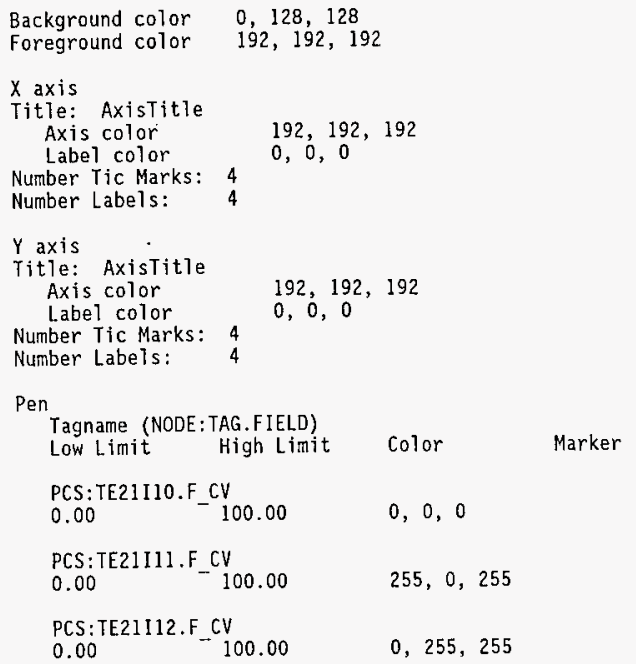

Time Duration $=00: 10: 00$

Object: PUSHBUTTON $186,607,1529,1007$

Command List

REPLACEPIC OFFGAS FURNOVER

Text: Furnace Overview

Command List

REPLACEPIC OFFGAS FURNOVER

Object: RECT $1340,4170,2680,4710$

Controllable: 0

Modifiable: 0

Object: LINE $\quad 2020,1740,2330,1800$ 
Dynamic Properties Report, OFFGAS.ODR

page 3

Controllable: 0

Modifiable: 0

Edge Color by value

Tagname: PCS:HS VAC2.F CV

$B 1$ ink on new alārm: $0^{-}$

$\begin{array}{lll}\text { Value } & \text { color } & \text { Property } \\ 0.00 & 0,0,0 & \text { VISIBLE } \\ 1 & 255,0,0 & \text { VISIBLE }\end{array}$

0bject: LINE $1665,1740,2025,1800$

Controllable: 0

Modifiable: 0

Edge Color by value

Tagname: PCS:HS VACl.F_CV

Blink on new alärm: $0^{-}$

$\begin{array}{lll}\text { Value } & \text { Color } & \text { Property } \\ 0.00 & 0,0,0 & \text { VISIBLE } \\ 1 & 255,0,0 & \text { VISIBLE }\end{array}$

Object: LINE $1970,1740,2030,4200$

Controllable: 0

Modifiable: 0

Edge color by value

Tagname: PCS:FAN ON.F CV

Blink on new alarm: $0^{-}$

$\begin{array}{lll}\text { Value } & \text { Color } & \text { Property } \\ 0.00 & 0,0,0 & \text { VISIBLE } \\ 1 & 255,0,0 & \text { VISIBLE }\end{array}$

Object: PUSHBUTTON $390,1560,1560,1970$

Command List

TOGGLEDIG PCS:HS VACI.F CV

Text: Vacuum Fan 1

Command List

TOGGLEDIG PCS:HS_VACl.F_CV

Object: PUSHBUTTON $2440,1560,3610,1980$ 
Dynamic Properties Report, OFFGAS.ODR

Command List

TOGGLEDIG PCS:HS_VAC2.F_CV

Text: Vacuum Fan 2

Command List

TOGGLEDIG PCS:HS_VAC2.F_CV

object: LINE.

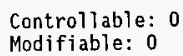

Controliable: 0

Modifiable: 0

Edge Color

by value

Tagname: PCS:HS VAC2, F CV

Blink on new alärm: 0

$$
\begin{aligned}
& \text { Value } \\
& 0.00 \\
& 1
\end{aligned}
$$

object: LINE

$1670,889,1730,1800$

Controllable: 0
Modifiable: 0

Edge Color by value

Tagname: PCS:HS_VAC1.F_CV

Blink on new alarm: $0^{-}$

Value Color

$0.00 \quad 0,0,0$

1 page 4

Property

VISIBLE

Object: LINE $\quad 1674,890,2330,950$

Controllable: 0

Modifiable: 0

Edge Color by value

Tagname: PCS:HS VAC1.F_CV

Blink on new alarm: 0

$\begin{array}{lll}\text { Value } & \text { Color } & \text { Property } \\ 0.00 & 0,0,0 & \text { VISIBLE } \\ 1 & 255,0,0 & \text { VISIBLE }\end{array}$

Object: LINE $\quad 2270,890,2880,950$ 
Dynamic Properties Report, OFFGAS.0DR

Controllable: 0

Modifiable: 0

Edge Color

by value

Tagname: PCS:FAN_ON,F_CV

B] ink on new alarm: $0^{-}$

$\begin{array}{lll}\text { Value } & \text { Color } & \text { Property } \\ 0.00 & 0,0,0 & \text { VISIBLE } \\ 1 & 255,0,0 & \text { VISIBLE }\end{array}$

Object: GROUP 285, 1211, 1784, 1541

Controllable: 0
Modifiable: 0

Foreground Color by value

Tagname: PCS:HS VAC1.F CV

Blink on new ajarm: 0

$\begin{array}{lll}\text { Value } & \text { Color } & \text { Property } \\ 0.00 & 0,0,0 & \text { VISIBLE } \\ 1 & 255,0,0 & \text { VISIBLE }\end{array}$

Object: GROUP 285, 1381, 1654, 1541

Controllable: 0

Modifiable: 0

Object: PGON 285, 1401, 1654, 1491

Controllable: 0

Modifiable: 0

Object: RECT $285,1471,1654,1541$

Controllable: 0

Modifiable: 0

Object: OVAL. $651,1381,708,1431$

Controliable: 0

Modifiable: 0

Object: RECT

$510,1381,586,1441$ page 5

VISIBLE 
Controllable: 0

Modifiable: 0

Object: RECT $388,1261,1775,1441$

Controllable: 0

Modifiable: 0

Object: GROUP $1297,1211,1784,1481$

Controllable: 0

Modifiable: 0

Object: GROUP $1503,1231,1784,1461$

Controllable: 0

Modifiable: 0

Object: RECT 1503,1231, 1654, 1461

Controllable: 0

Modifiable: 0

Object: RECT 1503, 1231, 1578, 1461

Controllable: 0

Modifiable: 0

Object: RECT $1644,1271,1784,1441$

Controllable: 0

Modifiable: 0

Object: RECT 1644, 1271, 1710, 1441

Controllable: 0

Modifiable: 0

Object: RECT 1297, 1211, 1513, 1481

Controllable: 0

Modifiable: 0

Object: OVAL $1455,1221,1513,1271$ 
Controllable: 0

Modifiable: 0

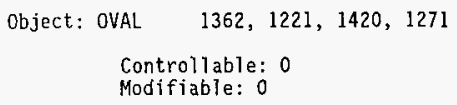

Object: OVAL $1306,1221,1364,1271$ Controllable: 0 Modifiable: 0

Object: OVAL $1409,1221,1467,1271$ Controllable: 0 Modifiable: 0

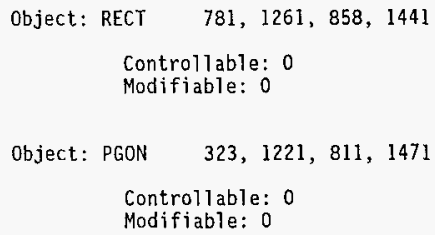

Object: OVAL $997,1391,1045,1441$ 
Controllable: 0

Modifiable: 0

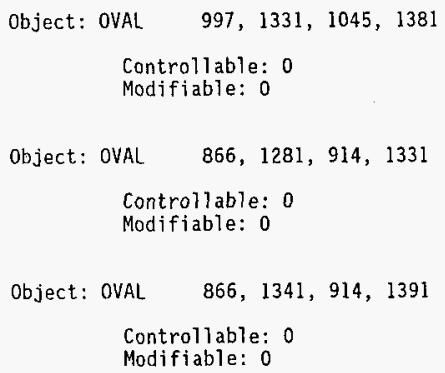


Controllable: 0

Modifiable: 0

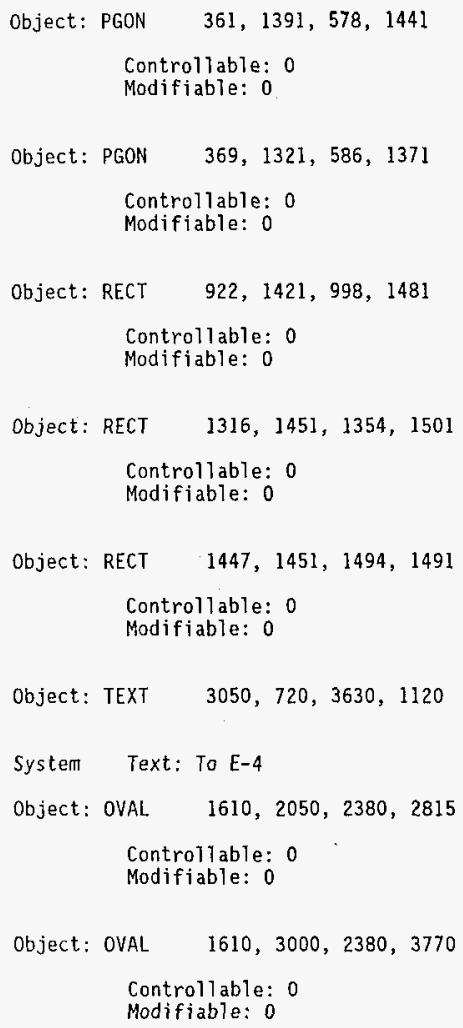

Object: TEXT $1720,3290,2330,3490$ 
Dynamic Properties Report, OFFGAS.00R

Text: Filter \#1

Object: TEXT 1710, 2350, 2320, 2550

Text: Filter \#2

Object: TEXT $\quad 1560,4240,2510,4640$

FurnacesText: From HA-2l I

Object: GROUP 2210,1211, 5363, 1541

Controllable: 0

Modifiable: 0

Foreground color by value

Tagname: PCS:HS VAC2.F CV

Blink on new alärm: $0^{-}$

Value Color

$0.00 \quad 0,0,0$

1 page 10

Property

VISIBLE

VISIBLE

Object: GROUP $\quad 2350,1381,5363,1541$

Controllable: 0

Modifiable: 0

Object: PGON 2350, 1401, 3810, 1491

Controllable: 0

Modifiable: 0

Object: RECT $2350,1471,3810,1541$

Controllable: 0

Modifiable: 0

Object: OVAl $\quad 3360,1381,3810,1431$

Controllable: 0

Modifiable: 0

Object: RECT $3490,1381,3810,1441$

Controllable: 0

Modifiable: 0 
WHC-SD-CP-CSWD-018. Rev. 0

Dynamic Properties Report, OFFGAS.0DR

page 11

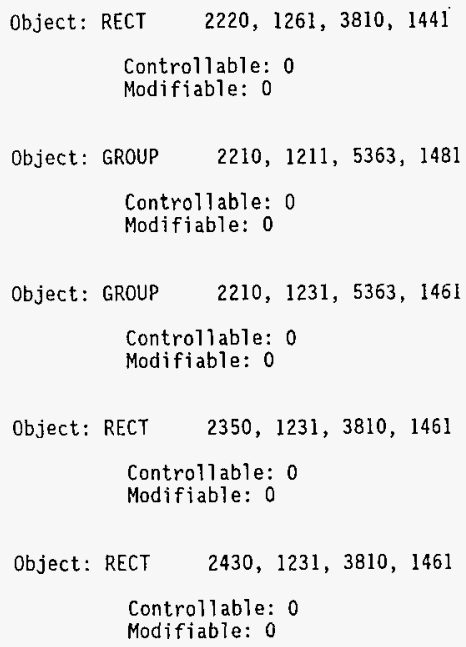

Object: GROUP $2210,1231,5363,1461$

Controllable: 0

Modifiable: 0

Object: RECT $2350,1231,3810,1461$

Controllable: 0

Modifiable: 0

Object: RECT $2430,1231,3810,1461$

Controllable: 0

Modifiable: 0

Object: RECT 2210, 1271, 3810, 1441

Controliable: 0

Modifiable: 0

Object: RECT 2290, 1271, 3810, 1441

Controllable: 0

Modifiable: 0

Object: RECT $2500,1211,3810,1481$

Controllable: 0

Modifiable: 0

Object: OVAL $\quad 2500,1221,3810,1271$

Controllable: 0

Modifiable: 0

Object: OVAL $2600,1221,3810,1271$ 
Controllable: 0

Modifiable: 0

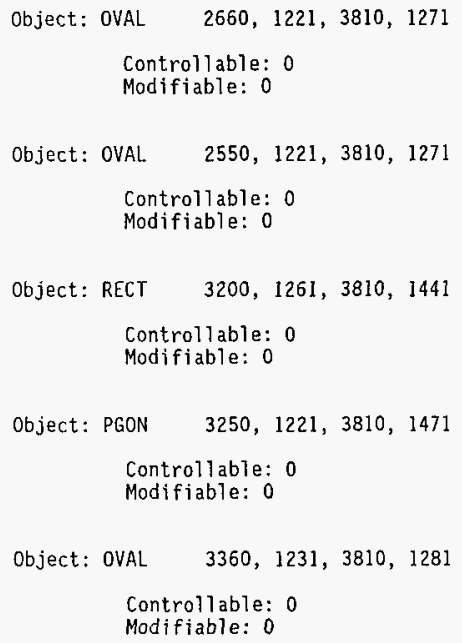

Object: GROUP $2990,1251,5363,1461$

Controllable: 0

Modifiable: 0

Object: RECT 2990, 1251, 3810, 1461

Controllable: 0

Modifiable: 0

Object: OVAL $3000,1391,3810,1441$

Controliable: 0

Modifiable: 0

Object: OVAL $3000,1331,3810,1381$ 
Controllable: 0

Modifiable: 0

Object: OVAL $\quad 3140,1281,3810,1331$

Controllable: 0

Modifiable: 0

Object: OVAL $3140,1341,3810,1391$

Controllable: 0

Modifiable: 0

Object: OVAL $3140,1391,3810,1441$

Controliable: 0

Modifiable: 0

Object: OVAL $3000,1281,3810,1331$

Controllable: 0

Modifiable: 0

Object: OVAL $\quad 3370,1331,3810,1381$

Controllable: 0

Modifiable: 0

Object: OVAL $3370,1281,3810,1331$

Controllable: 0

Modifiable: 0

Object: OVAL $3370,1381,3810,1431$

Controllable: 0

Modifiable: 0

Object: PGON $3480,1251,3810,1301$

Controllable: 0

Modifiable: a

Object: PGON $3500,1391,3810,1441$ 
Controllable: 0

Modifiable: 0

Object: PGON $3490,1321,3810,1371$

Controllable: 0

Modifiable: 0

Object: RECT $3050,1421,3810,1481$

Controllable: 0

Modifiable: 0

Object: RECT 2670, 1451, 3810, 1501

Controllable: 0

Modifiable: 0

Object: RECT 2520, 1451, 3810, 1491

Controllable: 0

Modifiable: 0

Object: TEXT $820,40,3320,350$

Text: Off-Gas Fan Control

Object: GROUP $\quad 4230,4100,7430,4760$

Controllable: 0

Modifiable: 0

Object: TEXT $4230,4110,7020,4310$

Text: FUR-21I-1 OFF-GAS TEMPERATURE

Object: TEXT 4240, 4330,7030, 4530

Text: FUR-21I-2 OFF-GAS TEMPERATURE

Object: TEXT 4240, 4540,7030, 4740

Text: FUR-21I-3 OFF-GAS TEMPERATURE

Object: DATALINK $7100,4100,7430,4320$ 
Dynamic Properties Report, OFFGAS.ODR

page 15

Controliable: 0

Modifiable: 0

Tagname: PCS:TE21I10.F_CV

Visible Background Colors: 255, 255, 255

Object: DATALINK $7090,4320,7420,4540$

Controllable: 0

Modifiable: 0

Tagname: PCS:TE21I11.F_CV

Visible Background Colors: 255, 255, 255

Object: DATALINK $7100,4540,7430,4760$

Controllable: 0

Modifiable: 0

Tagname: PCS:TE21I12.F_CV

Visible Background Colors: 255, 255, 255 
WHC-SD-CP-CSWD-018, Rev. 0

APPENDIX E

\section{TAG GROUP REPORTS}


WHC-SD-CP-CSWD-018, Rev. 0

The FIX DMACS Tag Group Report

File: C: WWDMACS\PIC\FUR21I1.TGR

November 6, 1996

\section{SUBSTITUTION}

PCS: FUR21I1.F CV

PCS:0P21I1.F $\bar{C} V$

PCS:TE21 IMAX.F CV

PCS:LS21I1.F CV

PCS:TE21I10.F CV

PCS:TE21I2.F $\overline{C V}$

PCS:TC 21I1. $\bar{F}$ CV

PCS:PWR $2111 . F^{-} C V$

PCS:PN21I1.F $\overline{C V}$

PCS:TP 21I1-C.F 2

PCS:SP-21I1.F $\mathrm{CV}^{-}$

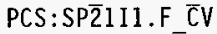

PCS:TP 2111-C.F_11

\section{DESCRIPTION}

FURNACE NUMBER

POWER TO FURNACE

HA-2OMB HIGH TEMPERATURE

DOOR LIMIT SWITCH

OFF-GAS TEMPERATURE

HIGH TEMPERATURE ALARM READING

FURNACE TEMPERATURE

FURNACE POWER

PROGRAM NUMBER

PGRM STATE $1=$ RESET 2=RUN

STOP FURNACE

FURNACE POINT

PROGRAM SEGMENT TIME REMAINING 
WHC-SD-CP-CSWD-018, Rev. 0

The FIX DMACS Tag Group Report

File: C: \WDMACS\PIC\FUR21I2. TGR November 6, 1996

\section{SYMBOL.}

FURNNAME

FURNPOWER

GBTEMP

LS

OFFGASTEMP

OVENHITEMP

OVENTEMP

POWER

PROGRAM

START

STOP

TEMPSETPOINT

TIMEREMAIN
SUBSTITUTION

PCS:FUR21I2.F CV

PCS:OP21I2.F CV

PCS:TE21 IMAX.F CV

PCS:LS21I2.F CV

PCS:TE21I11. $\bar{F} C V$

PCS:TE21I4.F $\bar{C} V$

PCS:TC $2112 . \bar{F}$ CV

PCS:PWR21 12. $\mathrm{F}^{-} \mathrm{CV}$

PCS:PN21I2.F CV

PCS:TP $2112-C . F 2$

PCS:SP $2112 . \bar{F} \mathrm{CV}^{-}$

PCS:SP $\overline{1} 112 . \bar{F} \overline{C V}$

PCS:TP_21I2_C.F_11

\section{DESCRIPTION}

FURNACE NUMBER

POWER TO FURNACE

HA-20MB HIGH TEMPERATURE

DOOR LIMIT SWITCH

HIGH TEMPERATURE ALARM READING

FURNACE TEMPERATURE

FURANCE POWER

PROGRAM NUMBER

START PRGM RESET $=1 \quad$ RUN $=2$

STOP FURNACE

FURNACE POINT

PROGRAM SEGMENT TIME REMAINING 
The FIX DMACS Tag Group Report

File: C: \WDMACS\PIC \FUR21I3.TGR November 6, 1996
SYMBOL
FURNNAME
FURNPOWER
GBTEMP
LS
OFFGASTEMP
OVENHITEMP
OVENTEMP
POWER
PROGRAM
START
STOP
TEMPSETPOINT
TIMEREMAIN

\section{SUBSTITUTION}

PCS:FUR21 I3.F CV

PCS:0P2113.F $\overline{\mathrm{C} V}$

PCS:TE21 IMAX.F CV

PCS:LS2113.F CV

PCS:TE21I12. $\bar{F}$ CV

PCS:TE21I6.F $\overline{C V}$

PCS:TC $2113 . \overline{\mathrm{F}} \mathrm{CV}$

PCS: PWR 21I3. F $\mathrm{CV}$

PCS: PN21 I3. F $\bar{C} V$

PCS:TP 2113 - C.F 2

PCS:SP $2113 . \bar{F} C V$

PCS:SPर̄1I3.F $\bar{C} V$

PCS:TP 2113-C.F 11
DESCRIPTION

POWER TO FURNACE

HA-2OMB HIGH TEMPERATURE

DOOR LIMIT SWITCH

OFF-GAS TEMPERATURE

HIGH TEMPERATURE ALARM READING

FURNACE TEMPERATURE

FURNACE POWER

PROGRAM NUMBER

START PRGM RESET $=1 \quad$ RUN $=2$

STOP FURNACE

FURNACE POINT

PROGRAM SEGMENT TIME REMAINING 